\title{
Indice acumulativo del Boletín de la Sociedad Botánica de México de los números 41 (1981) al 50 (1990).
}

\begin{abstract}
ARMANDO BUTANDA ${ }^{1}$
Ofrecer fuentes de información a la inquietud de los lectores, cuando tienen un fundamento científico honra a quien las ofrece y aprovecha a quien las adquiere (Anónimo).
\end{abstract}

\section{INTRODUCCIÓN}

El propósito de este índice acumulativo al igual que el publicado en 1981 (números $1 \mathrm{al}$ 40) es el de facilitar la consulta de todos los artículos científicos aparecidos y publicados por la Sociedad Botánica de México, A.C. en sus boletines números 41 al 50, años 1981 a 1990.

Siendo estos boletines exponentes de la investigación botánica en México y con su contenido se ha difundido ampliamente contribuciones al conocimiento de los recursos vegetales. Por lo que con la publicación del presente Indice se pretende satisfacer la necesidad que tiene el lector en obtener la información acumulada en ellos de una manera rápida y fácil, ya que en los años que se abarcan comprende 10 números, 118 artículos y 126 autores.

Para el arreglo de este índice acumulativo se siguió el mismo formato usado en la versión de 1981. Dividido en tres partes: la primera consiste en una lista en orden cronológico de los trabajos firmados, la segunda es un índice integrado por las palabras clave de los títulos de los trabajos en orden alfabético incluyendo los autores, la tercera es un índice de taxa y temas que incluye en el caso de los taxa, los aparecidos en el título o en el texto y para los nombres vulgares su equivalente científico, así como también los nuevos taxa para la ciencia y los cambios nomenclaturales. La entrada principal es por familias de las plantas o los animales, excepto para las criptógamas que es por grupos (Pteridophyta, Bryophyta, Fungi, Algae). Cuando los títulos se refieren a una localidad geográfica tienen una entrada por país, estado o región.

${ }^{1}$ Departamento de Botánica, Instituto de Biología, Universidad Nacional Autónoma de México, Apdo. Postal 70-233, Delegación Coyoacán 04510, México, D. F.

Butanda-Cervera A. 1990. Indice acumulativo del Boletín de la Sociedad Botánica de México de los números 41 (1981) al 50 (1990). Boletín de la Sociedad Botánica de México 50: 167-214. 


\section{INDICE CRONOLOGICO}

Boletín Núm. 41, noviembre, 1981.

Productividad primaria de tres praderas de especies tropicales: pará (Brachiaria mutica), grama amarga (Paspalum conjugatum) y pangola (Digitaria decumbens), Ricardo Almeida $M \cdot 2.18$

Una nueva especie de Tauschia (Umbelliferae) del Estado de México, Graciela Calderón de Rzedowski y Lincoln Constance:19-21

Nueva especie de Casimiroa (Rutaceae) de la zona árida oaxaqueñopoblana, Fernando Chiang y Francisco González-Medrano:23-26

Adiciones a la flora arvense del Valle de México, Francisco Javier Espinosa García:27-32

Distribución esporangial en estróbilos de especies de Selaginella, Ma. Eugenia Frayle y Ramón Riba:33-40

Rzedowskia, un nuevo género de Celastraceae de México, F. GonzálezMedrano:41-46

Sobre la ecología reproductiva de Nicotiana glauca Grah: Una maleza de distribución cosmopolita, Héctor Manuel Hernández:47-73 Estructura y composición la vegetación herbacea de un bosque uniespecífico de Pinus hartwegii I.

Estructura y composición florística, Ma. Cristina Obieta y José Sarukhán:75125

Vegetation and flora of the Bolson of Cuatro Cienegas region, Coahuila, Mexico: III Cactaceae to Compositae, Donald J. Pinkava:127-151
Ormosia (Leguminosae) in Mexico, including a new species from Oaxaca, Velva E. Rudd:153-159

\section{Notas Botánicas}

Nuevo registro para México de Pontederia rotundifolia, Alejandro Novelo Retana:161

Rizophora harrisonii (Rhizophoraceae), un nuevo registro para las costas de México, Víctor Rico Gray:163-165

Nota sobre la distribución de Carpodiptera ameliae (Tiliaceae), en México, Oswaldo Téllez Valdez:166

Nota aclaratoria sobre las hojas de Valeriana vaginata (Valerianaceae), Rosa Ma. Vega y J. Rzedowski:167-170

Boletín Núm. 42, agosto, 1982.

Componentes fenológicos del sistema de cruzamiento monoico de Cnidoscolus spinosus (Euphorbiaceae) en Jalisco, Stephen H. Bullock:1-9

The impact of forest thinning on microclimate in monarch butterfly (Danaus plexippus L.) overwintering areas of Mexico, William H. Calvert, Willow Zuchowski y Lincoln P. Brower:1118

Comparative photosynthetic characteristics of coastal and desert plants of California, H.A. Mooney, J. Berry, O. Björkman y J. Ehleringer:19-33

Jatropha chamelensis (Euphorbiaceae), nueva especie de la costa de 
Jalisco, México, L. Alfredo PérezJiménez:35-39

Estudio de los canales resiníferos de la corteza de Bursera copallifera y Bursera grandifolia, Guadalupe Suárez Ramos y E. Mark Engleman:41-54

The altitudinal distribution of the genus Pinus in the Western United States and Mexico, Richard I. Yeaton:55-71

Guía para los autores a partir del número 43:73-75

Boletín Núm. 43, diciembre, 1982.

Una nueva especie de Decazyx (Rutaceae) de Tabasco y Veracruz, Fernando Chiang:1-3

Nuevas combinaciones y una nueva variedad de Lycium (Solanaceae) de Norteamérica, Fernando Chiang:5-8

Estudios cromosómicos en Lycium (Solanaceae) de Norteamérica, Fernando Chiang:9-23

Estudio del polen de Phaseolus chiapasanus Piper (Leguminosae: Phaseolinae), Alfonso Delgado Salinas, Enrique Martínez Hernández y Pilar Fernández Ortuño:25-34

Musgos de la Península de Yucatán, México. II., Claudio Delgadillo y Angeles Cárdenas:35-37

Mimosa martindelcampoi Medrano (Legunimosae), una specie nueva del sudoeste de Tamaulipas, México, Francisco González Medrano:39-42

Aspectos ecológicos de Mimosa biuncifera y Mimosa monancistra en el noroeste del estado de Guanajuato, Rosaura Grether:43-60

Neoeplingia Ramamoorthy, Hiriart Medrano (Labiatae) un nuevo género de
Hidalgo, México, T.P. Ramamoorthy, P. Hiriart Valencia y F. González Medrano:61-65

Acacia velvae $y$ A. mirandae (Leguminosae), dos nuevas especies para México, Ma. de Lourdes Rico Arce:67-71

Estudios quimiotaxonómicos de Bursera (Burseraceae).I. Bursera chemapodicta sp. n., Jerzy Rzedowski y Evangelina Ortiz:73-80

\section{Notas Botánicas}

Un aparato sencillo para medir la dureza del follaje en estudios de herbivoría, Rodolfo Dirzo, Adrián Fernández Bremauntz, Gabriela Gómez Rodríguez y Verónica González Kladiano:81-88

$\mathrm{El}$ banco de esporas de helechos en el suelo de los Tuxtlas, Ver., B. PérezGarcía, A. Orozco-Segovia y R. Riba:8992

\section{Reseña de Libros}

Flora of Chiapas. Part 2 Pteridophytes, Alan R. Smith. California Academy of Sciences, 1981, Ramón Riba:93

Ecología de la Estación Experimental Zoquiapan (descripción general, vegetación y fauna). Blanco Z.S. y otros. UACH, 1978, Martha Pérez García:94

Plantas medicinales del estado de Yucatán. Mendieta, R.M. y S. del Amo R. CECSA, 1981, Alma OrozcoSegovia:94-95

Estudios ecológicos en el agroecosistema cafetalero. Jiménez 
Avila y A. Gómez-Pompa (Edrs.), CECSA, 1982, Tere Chehaibar Nader:95

Tipos de vegetación y su distribución en el estado de Tabasco y norte de Chiapas. Mendoza, R.L. UACH, 1980, Alma Orozco Segovia:96

Guía para los autores:97-99

Boletín Núm. 44, diciembre, 1983.

Los géneros de Convolvulaceae en México, D.F. Austin y R.A. Pedraza:3-16

Nuevos registros de ciperáceas para la flora del Valle de México y de la República Mexicana, Ma. Socorro González E.:17-21

Selaginella rzedowskii, una nueva especie de selaginela heterófila del estado de Guerrero, México, Francisco G. Lorea-Hernández:23-27

El polen del género Agave para la Península de Yucatán, Beatriz LudlowWiechers y Lina Ojeda:29-42

Helechos de Veracruz: Adiantum (Pteridaceae), Mónica Palacios-Ríos y Ramón Riba:43-62

Análisis de la interacción entre radiaciones gamma y $\mathrm{mms}$ sobre la plántula de semillas de Triticale pretratadas con ácido ascórbico, Guadalupe Palomino Hasbach:63-71

Dos nuevas especies mexicanas de la familia Rubiaceae, J. Rzedowski:73-80

Plantae uxpanapae I. Colubrina johnstonii sp. nov. (Rhamnaceae), Tom Wendt:81-90

\section{Notas Botánicas}

Sobre el significado del "co" en coevolución, Rodolfo Dirzo:91-94

Nota sobre tres especies de ciperáceas del Valle de México, Socorro González E.:95-96

Notas sobre la fitogeografía del bosque mesófilo de montaña en la Sierra Madre del Sur, México, S-A, Lorenzo, Lila, A. Ramírez Roa, M.A. Soto Arenas, A. Breceda, M. del Calderón, H. Cortéz, C. Puchet, M. Ramírez, R. Villalón y E. Zapata:97-102

\section{Reseña de Nuevos Libros}

Ferns and allied plants with special reference to tropical américa. Rolla $\mathrm{M}$. Tryon y A.F. Tryon, Harvard University 1982, Ramón Riba:103-104

Colonización y destrucción de bosques en Panamá. Heckadon Moreno, S. y A. McKay (Edrs.), Asociación Panameña de Antropología, 1982, Tere Chehaibar Nader:104-105

El peregrinar de las flores mexicanas. José Mariano Mociño y Lozada 1757-1822, P. R. Grobet, INIREB, CECSA, 1982, Nidia Pérez Naser:105-106

Boletín Núm. 45, diciembre, 1983. (fecha de publicación julio, 1984).

Detalles ultraestructurales de la madera de algunas Boraginaceae de México, Josefina Barajas Morales:3-14

Notas taxonómicas y observaciones sobre algunas especies mexicanas $d$. 
Gnaphalium (Compositae), Francisco Javier Espinosa-García:15-21

Vegetación insular de la Península de Yucatán, José Salvador Flores Guido:23-37

Algunos aspectos ecológicos y fitogeográficos de las especies de Cyperaceae en el Valle de México. I. Afinidades ecológicas, Socorro González E. y J. Rzedowski:39-47

Análisis de la vegetación arbórea en la provincia florística de TehuacánCuicatlán, Víctor Jaramillo Luque y Francisco González Medrano:49-64

First record of Elaeagia (Rubiaceae) in Mexico, with description of a new species, E. uxpanapensis, David $\mathrm{H}$. Lorence:65-69

Estudio demográfico del lirio acuático Eichhornia crassipes (Mart) Solms: dinámica de crecimiento en dos localidades selectas de México, Martha S. Niño Sulkowska y Antonio Lot:71-83

Esclarecimiento taxonómico de Nymphaea gracilis Zucc., planta acuática endémica de México, Alejandro Novelo R. y Antonio Lot H.:85-95

Algunas adiciones al género Acourtia (Compositae - Mutisieae), J. Rzedowski:97-109

Dos especies nuevas de CompositaeAstereae del Valle de México, J. Rzedowski:111-116

Características palinológicas de las araliáceas de México, Victoria Sosa:117132

Plantae uxpanapae II. Novedades en Violaceae y Scrophulariaceae, Tom Wendt:133-140

\section{Notas Botánicas}

El número cromosómico de Grabowskia geniculata (Solanaceae), Fernando Chiang: 141-142

Condalia velutina (Rhamnaceae) una planta muy poco colectada, Rafael Fernández Nava:143-146

Registros nuevos de plantas acuáticas mexicanas I: Myriophyllum quitense H.B.K. (Haloragaceae), Alejandro Novelo Retana:147-149

Especies CAM en la selva húmeda tropical de Los Tuxtlas, Veracruz, Clara Tinoco Ojanguren y Carlos VázquezYanes:150-153

Boletín Núm. 46, junı, 1984.

(fecha de publicación junio, 1986)

Biología floral de poblaciones silvestres y cultivadas de Phaseolus coccineus L. II. Sistemas reproductivos, Alberto Búrquez y José Sarukhán:3-12

Musgos de Zacatecas, México II, Angeles Cárdenas S. y Claudio Delgadi1lo M.:13-18

Rango de hospedantes de Rhynchosporium secalis, Magda Carvajal Moreno:19-24

Nuevos registros de Juncaceae y Liliaceae en el Valle de México, Raquel Galván Villanueva:25-27

Algunos aspectos ecológicos y fitogeográficos de las especies de Cyperaceae en el Valle de México II. Análisis fitogeográfico, Socorro González E. y J. Rzedowski: 29-35

Una especie nueva de Stegnosperma (Phytolaccaceae) del suroeste de Puebla, 
México, Francisco González-Medrano y Rosalinda Medina Lemos:37-41

Una nueva especie de Scutellaria (Labiatae) de Hidalgo, México, Patricia Hiriart Valencia:43-46

Un género nuevo de la parte meridional de México: Gypsacanthus (Acanthaceae, Justicieae, Odontoneminae), Emily J. Lott, Víctor Jaramillo L. y Jerzy Rzedowski:47-51

Interpretaciones sobre la lluvia de polen en la región de San Luis Potosí, México, Socorro Lozano-García:53-74

La vegetación acuática vascular de seis lagos-cráter del estado de Puebla, México, Pedro Ramírez-García y Alejandro Novelo R.:75-88

\section{Notas Botánicas}

Nota sobre la ortografía correcta de Casimiroa Calderonii Chiang et Medrano, Fernando Chiang:89

Registros nuevos de plantas acuáticas mexicanas

.II Luziola subintegra Swallen y $\ddot{L}$. spruceana Benth. ex Doell. (Gramineae), Alejandro Novelo R.:9091

\section{Reseña de Libros}

Malezas de Buenavista, Coahuila, José A. Villarreal Q., UAAAN, 1983, Francisco J. Espinosa García:93

Boletín Núm. 47, julio, 1987.(fecha de publicación 10 de julio, 1987)

Tigridia martinezii, una especie nueva de iridáceas del estado de Hidalgo
(México), Graciela Calderón de Rzedowski:3-6

Musgos alpinos del Nevado de Colima, México, Claudio Delgadillo M.:7-11

Musgos de Zacatecas, México III. Síntesis y fitogeografía,Claudio Delgadillo M. y Angeles Cárdenas S.:13-24

Notas sobre la ecología reproductiva de árboles en un bosque mesófilo de montaña en Michoacán, México, Héctor M. Hernández y Yasmín Carreón Abud:25-35

New taxa and a new name in Mexican and Central American Randia (Rubiaceae, Gardenieae), David H. Lorence y John D Dwyer: 37-48

New taxa in Mexican Psychotria (Rubiaceae, Psychotrieae), David H. Lorence y John D. Dwyer: 49-64

Clave genérica para las compuestas de la cuenca del río Balsas, José Luis Villaseñor:65-86

\section{Notas Botánicas}

Nota sobre la identidad del segundo Tzonpotónic mencionado por Francisco Hernández en su Historia Natural de la Nueva España, Francisco Javier Espinosa García:90-91

Nota sobre la distribución de Savia sessiliflora (Euphorbiaceae) en México, Emilly J. Lott:90-91

Lectotipificación de Limnocharis laforestii Duchass. ex Griseb. (Limnocharitaceae), Alejandro Novelo R.:9293 


\section{Reseña de Libros}

Flora fanerogámica del Valle de México, volumen II, Dicotyledonàe (Euphorbiaceae-Compositae). Jerzy Rzedowski y Graciela C. de Rzedowski, IPN e Instituto de Ecología, 1985, José Luis Villaseñor: 95-96

Boletín Núm. 48, diciembre, 1988. 1989).

(fecha de publicación 10 de mayo,

Distribución de Rhynchosporium secalis en el mundo y en México, Magda Carvajal Moreno:3-11

Algunos musgos de Tabasco, México, Claudio Delgadillo M. y Sergio Zamúdio:13-18

Nuevos registros y nuevas combinaciones de gramíneas del Valle de México, Yolanda Herrera A.:19-22

Allosidastrum, un nuevo género de Malvaceae de los neotrópicos, Antonio Krapovickas, Paul A. Fryxell y David M. Bates:23-24

Reconocimiento florístico y consideraciones fitogeográficas del bosque mesófilo de montaña de Teocelo, Veracruz, Isolda Luna, Lucía Almeida, Lourdes Villers y Lila Lorenzo:35-63

Métodos computarizados y algunas de sus aplicaciones al estudio de la flora de México, Nancy P. Moreno y Robert Allkin:65-73

Estudios citogenéticos de dos especies y una variedad del género $\mathrm{Nyc}$ tocereus (Cactaceae), Guadalupe Palomino Hasbach, Socorro Zuleta Lechuga y Leia Scheinvar:75-80
Inventario florístico en el cerro Tetzcotzinco, Texcoco, Estado de México, Ma. Teresa P. Pulido y Stephen D. Koch: 81-94

Estudio biosistemático de Eragrostis mexicana, E. neomexicana, E. orcuttiana y E. virescens (Gramineae: Chloridoideae), Isidoro Sánchez Vega y Stephen D. Koch:95-112

Water contents of wood of tropical deciduous forest species during the dry season, Ernst-Detlef Schulze, Harold A. Mooney, Stephen H. Bullock, y Ana Mendoza:113-118

El maguey (Agave, subgénero Agave) en el altiplano potosinozacatecano, J. Jesús Tello Balderas y Edmundo García Moya:119-134

Spigelia longiflora (Mart. et. Gal.): actividad antialimentaria en tres especies de insectos fitófagos, Miguel Angel Villavicencio y Blanca E. Pérez Escandón:135-138

\section{Notas Botánicas}

Notas sobre las perturbaciones naturales en un bosque mesófilo de montaña en Tamaulipas, Laura Arriaga:139-142

Nuevo registro para México de Gleditsia (Leguminosae), Oscar L. Briones V::143-144

El número cromosómico de Lycium leiospermum I. M. Johnston (Solanaceae), Fernando Chiang:145-146

Reducción en la fecundidad en Manfreda brachystachya (Cav.) Rose, una agavácea polinizada por murciélagos: los riesgos de la 
especialización en la polinización, Luis E. Eguiarte y Alberto Búrquez:147-149

Nota sobre la identidad del tepescohuite en México, Rosaura Grether:151-152

Consideraciones sobre la exina en Yucca, observada al microscopio de luz y microscopio de barrido, Rodolfo Palacios-Chávez, Beatriz LudlowWiechers y Lina Ojeda:153-154

Una adición al género Ormosia (Leguminosae) en México: O.panamensis, Velva E. Rudd y Tom Wendt:155-158

\section{Reseña de Libros}

Bibliografía comentada sobre pteridófitas de México, Ramón Riba y Armando Butanda, Consejo Nacional de la Flora de México, 1987, María de la Luz Arreguín-Sánchez:159

Boletín Núm. 49, diciembre, 1989.

La fisiología ecológica de plantas, Carlos Vázquez-Yanes:3-5

Nutrición mineral, Emanuel Rincón y Pilar Huante:7-17

Aspectos ecofisiológicos de las micorrizas, María Valdés: 19-30

El papel del microclima en la fisiología ecológica vegetal, Víctor L. Barradas:31-39

Fotosíntesis y economía del carbono en plantas superiores, Alejandro E. Castellanos V.:41-60

El almacenamiento de semillas en la conservación de especies vegetales. Problemas y aplicaciones, Carlos Vázquez-Yanes y Jorge R. Toledo:61-69
Fisiología y ecología del fitocromo: su función en las semillas, Alma OrozcoSegovia: $71-84$

Papel de los aleloquímicos en el manejo de los recursos naturales, Ana Luisa Anaya Lang:85-98

Boletín Núm. 50, diciembre, 1990.

Anatomía de la madera del género Mimosa (Leguminosae), Teresa Chehaibar y Rosaura Grether: 3-17

Lista florística de las algas marinas bentónicas de la costa norte de Michoacán, México, Kurt M. Dreckmann, Francisco F. Pedroche y Abel Sentíes G.: 19-42

La familia Rubiaceae en la región de Los Tuxtlas, Veracruz, México, David H. Lorence y Guillermo IbarraManríquez: 43-68

Parasitismo floral por abejas sociales (Meliponinae; Apidae) en el árbol quiropterófilo Crescentia alata (Bignoniaceae), Carlos Martínez del Río y Stephen H. Bullock: 69-76

Estudios cromosómicos en seis especies mexicanas de comelináceas, Guadalupe Palomino Hasbach, Balbina Vázquez Benitez, Patricia Martínez Almeraya y Pedro Mercado Ruaro:77-87

La familia Rhodomelaceae (Ceramiales, Rhodophyta) en la costa del estado de Michoacán, Abel Sentíes G., Francisco F. Pedroche y Kurt M. Dreckmann: 89-120

Caracterización ficoflorística de los paredones de la Sierra de Juárez, Oaxaca. Importancia de las formas de crecimiento algales en la tipificación de 
un ambiente, Rosa Luz Tavera-Sierra y Jorge González-González:121-133

Fitogeografía del Valle de Tehuacán-Cuicatlán, José Luis Villaseñor, Patricia Dávila y Fernando Chiang:135-149

\section{Notas Botánicas}

La preservación con alcohol de ejemplares colectados para herbario, Juan Ismael Calzada y Hugo Perales Rivera:151-155

Nota sobre la propagación y pérdida de viabilidad de las semillas de Chiranthodendron pectadactylon Larr. (Sterculiaceae), José Guadalupe García-Franco y Hugo R. Perales Rivera: 157-159

\section{Reseña de Libros}

Contribuciones de Eizi Matuda (1894-1978) al conocimiento de la flora de México, por Armando Butanda, Universidad Nacional Autónoma de México, México, 1989, 67p. (Cuadernos del Instituto de Biología 1). ISBN 968-36-11036, Ramón Riba:161

Pteridophyte flora of Oaxaca, México, por John T. Mickel y Joseph M. Beitel, Memoirs of the New Botanical Garden 46:1-569, 129 figs., 1988, ISBN 0-89327-323-6, Ramón Riba:161-162

A neotropical companion: an introduction to the animals, plants and ecosystems of the New World tropics. By John C. Kricher. Princeton University Press, Princeton, New Jersey, 1989, 436p., Rodolfo Dirzo: 163-165

\section{Indice acumulativo}

Indice acumulativo del Boletín de la Sociedad Botánica de México de los números 41 (1981) al 50 (1990), Armando Butanda:167-214 


\section{INDICE DE AUTORES Y TITULOS}

Abejas sociales (Meliponinae; Apidae) en el árbol quiropterófilo Crescentia alata (Bignoniaceae), Parasitismo floral por, 50: 69-76

Acacia velvae $y$ A. mirandae (Leguminosae), dos nuevas especies para México, 43:67-71

Acanthaceae, Justicieae, Odontoneminae, Un género nuevo de la parte meridional de México: Gypsacanthus, 46:47-51

Acido ascórbico, Análisis de la interacción entre radiaciones gamma y mms sobre la plántula de semillas de Triticale pretratadas con, 44:63-71 Acourtia (Compositae-Mutisieae), Algunas adiciones al género, 45:97-109 Actividad antialimentaria en tres especies de insectos fitófagos, Spigelia longiflora (Mart. et. Gal.):, 48:135-138 Acuática endémica de México, Esclarecimiento taxonómico de $\mathrm{Nym}$ phaea gracilis Zucc., planta, 45:85-95 Acuáticas mexicanas I: Myriophyllum quitense H.B.K. (Haloragaceae), Registros nuevos de plantas, 45:147149;II Luziola subintegra Swallen y $L$. spruceana Benth. ex Doell. (Gramineae), 46:90-91.

Adiantum (Pteridaceae), Helechos de Veracruz, 44:43-62

Adición al género Ormosia (Leguminosae) en México: $O$. panamensis, Una, 48:155-158

Adiciones a la flora arvense del Valle de México, 41:27-32

Afinidades ecológicas, Algunos aspectos ecológicos y fitogeográficos de las especies de Cyperaceae en el Valle de México. I., 45:39-47

Agavácea polinizada por murciélagos: los riesgos de la especialización en la polinización, Reducción en la fecundidad en Manfreda brachystachya (Cav.) Rose, una, 48:147-149 Agave para la Península de Yucatán, El polen del género, 44:29-42 Agave, subgénero Agave en el altiplano potosino-zacatecano, El maguey, 48:119-134

Aleloquímicos en el manejo de recursos naturales, Papel de los, 49:85-98

Algas marinas bentónicas de la costa norte de Michoacán, México, Lista florística de las, 50: 19-42

Algunas adiciones al género Acourtia (Compositae-Mutisieae), 45:97-109 Algunos aspectos ecológicos y fitogeográficos de las especies de Cyperaceae en el Valle de México I. Afinidades ecológicas, 45:39-47; II. Análisis fitogeográfico, 46:29-35

Algunos musgos de Tabasco, México, 48:13-18

ALLKIN, ROBERT Y NANCY P. MORENO. Métodos computarizados y algunas de sus aplicaciones al estudio de la flora de México, 48:65-73

Allosidastrum, un nuevo género de Malvaceae de los neotrópicos, 48:23-34 Almacenamiento de semillas en la conservación de especies vegetales. Problemas y aplicaciones, El, 49:61-69 ALMEIDA, LUCIA, ISOLDA LUNA, LOURDES VILLERS y LILA 
LORENZO. Reconocimiento florístico y consideraciones fitogeográficas del bosque mesófilo de montaña de Teocelo, Veracruz, 48:35-63

ALMEIDA M., RICARDO. Productividad primaria de tres praderas de especies tropicales: pará (Brachiaria mutica), grama amarga (Paspalum conjugatum) y pangola (Digitaria decumbens), 41:3-18

Altiplano potosino-zacatecano, El maguey (Agave, subgénero Agave) en $\mathrm{el}, 48: 119-134$

Altitudinal distribution of the genus Pinus in the Western United States and Mexico, The, 42:55-71

Análisis de la interacción entre radiaciones gamma y $\mathrm{mms}$ sobre la plántula de semillas de Triticale pretratadas con ácido ascórbico, 44:63-71

Análisis de la vegetación arbórea en la provincia florística de TehuacánCuicatlán, 45:49-64

Análisis fitogeográfico, Algunos aspectos ecológicos y fitogeográficos de las especies de Cyperaceae en el Valle de México II, 46:29-35

Anatomía de la madera de algunas especies del género Mimosa (Leguminosae), 50:3-17

ANAYA LANG, ANA LUISA. Papel de los aleloquímicos en el manejo de los recursos naturales, 49:85-98

Aparato sencillo para medir la dureza del follaje en estudios de herbivoría, Un, 43:81-88

Aplicaciones al estudio de la flora de México, Métodos computarizados y algunas de sus, 48:65-73
Araliáceas de México, Características palinológicas de las, 45:117-132

Arbol quiropterófilo Cresentia alata (Bignoniaceae), Parasitismo floral por abejas sociales (Meliponinae; Apidae) en el, 50:69-76

Arboles en un bosque mesófilo de montaña en Michoacán, México, Notas sobre la ecología reproductiva de, 47:25-35

ARREGUIN-SANCHEZ, MARIA DE LA LUZ. Reseña de libros, 48:159

ARRIAGA, LAURA. Notas sobre las perturbaciones naturales en un bosque mesófilo de montaña en Tamaulipas, 48:139-142

Aspectos ecofisiológicos de las micorrizas, 49:19-30 Aspectos ecológicos de Mimosa biuncifera y Mimosa monancistra en el noroeste del estado de Guanajuato, 43:43-60

Astereae del Valle de México, Dos especies nuevas de Compositae, 45:111-116

AUSTIN, D.F. y R.A. PEDRAZA. Los géneros de Convolvulaceae en México, 44:3-16 Banco de esporas de helechos en el suelo de Los Tuxtlas, Ver., Un, 43:89-92

BARAJAS MORALES, JOSEFINA. Detalles ultraestructurales de la madera de algunas Boraginaceae de México, 45:3-14

BARRADAS, VICTOR L. El papel del microclima en la fisiología ecológica vegetal, 49:31-39

BATES, DAVID M., ANTONIO KRAPOVICKAS y PAUL A. FRYXELL. Allosidastrum, un nuevo género de Malvaceae de los neotrópicos, 48:23-34 
BERRY, O. BJORKMAN, J. EHLERINGER y H.A. MOONEY. Comparative photosynthetic characteristics of coastal and desert plants of California, 42:19-33

Bignoniaceae, Parasitismo floral por abejas sociales (Meliponinae; Apidae) en el árbol quiropterófilo Crescentia alata, 50:69-76

Biología floral de poblaciones silvestres y cultivadas de Phaseolus coccineus L. III. Sistemas reproductivos, 46:3-12

Biosistemática de Eragrostis mexicana, $E$. neomexicana, $E$. orcuttiana y $E$. virescens (Gramineae: Chloridoideae), 48:95-112

BJORKMAN, O., J. EHLERINGER, H.A. MOONEY y J. BERRY. COMparative photosynthetic characteristics of coastal and desert plants of California, 42:19-33

Boletín de la Sociedad Botánica de México de los números 41 (1981) al 50 (1990), Indice acumulativo del, 50:167 $-214$

Bolson of Cuatro Cienegas region, Coahuila, Mexico: IIII Cactaceae to Compositae, Vegetation and, flora of the, $41: 127-151$

Boraginaceae de México, Detalles ultraestructurales de la madera de algunas, 45:3-14

Bosque mesófilo de montaña de Teocelo, Veracruz, Reconocimiento florístico y consideraciones fitogeográficas del, 48:35-63

Bosque mesófilo de montaña en Michoacán, México, Notas sobre la ecología reproductiva de árboles en un, 47:25-35
Bosque mesófilo de montaña en Tamaulipas, Notas sobre las perturbaciones naturales en un, 48:139-142 Bosque mesófilo de montaña en la Sierra Madre del Sur, México, Notas sobre la fitogeografía del, 44:97-102

Bosque uniespecífico de Pinus hartwegii

Estructura y composición florística, Estructura y composición la vegetación herbácea de un, 41:75-125 Brachiaria mutica, grama amarga (Paspalum conjugatum) y pangola (Digitaria decumbens), Productividad primaria de tres praderas de especies tropicales:pará, 41:3-18

BRECEDA, A., M. DEL C. CALDERON, H. CORTEZ, C. PUCHET, M. RAMIREZ, R. VILLALON, E. ZAPATA, LILA LORENZO, A. RAMIREZ ROA y M.A. SOTO ARENAS.Notas sobre la fitogeografía del bosque mesófilo de montaña en la Sierra Madre del Sur, México. 44:97-102

BRIONES V., OSCAR L. Nuevo registro para México de Gleditsia. (Leguminosae), 48:143-144

BROWER, LINCOLN P., WILLIAM H. CALVERT y WILLOW ZUCHOWSKI. The impact of forest thinning on microclimate in monarch butterfly (Danaus plexippus L.) overwintering areas of Mexico, 42:11-18

BULLOCK, STEPHEN H. Componentes fenológicos del sistema de cruzamiento monoico de 'Cnidoscolus spinostus (Euphorbiaceae) en Jalisco, 42:1-9

BULLOCK, STEPHEN H. y CARLOS MARTINEZ DEL RIO. Parasitismo floral por abejas sociales 
(Meliponinae; Apidae) en el árbol quiropterófilo Crescentia alata (Bignoniaceae), 50: 69-76

BULLOCK, STEPHEN H., ERNSTDETLEF SCHULZE, HAROLD A. MOONEY y ANA MENDOZA. Water contents of wood of tropical deciduous forest species during the dry season, 48:113-118

BURQUEZ, ALBERTO y JOSE SARUKHAN. Biología floral de poblaciones silvestres y cultivadas de Phaseolus coccineus L. II. Sistemas reproductivos, 46:3-12

BURQUEZ, ALBERTO y LUIS E. EGUIARTE. Reducción en la fecundidad en Manfreda brachystachya (Cav.) Rose, una agavácea polinizada por murciélagos: los riesgos de la especialización en la polinización, 48:147-149

Bursera (Burseraceae). I. Bursera chemapodicta sp. n., Estudios quimiotaxonómicos de, 43:73-80

Bursera chamapodicta sp. nov. Estudios quimiotaxonómicos de Bursera (Burseraceae). I., 43:73-80

Bursera copallifera y Bursera grandifolia, Estudio de los canales resiníferos de la corteza de, 42:41-54

Bursera grandifolia, Estudio de los canales resiníferos de la corteza de Bursera copallifera y, 42:41-54

BUTANDA, ARMANDO. Indice acumulativo del Boletín de la Sociedad Botánica de México de los números 41 (1981) al 50 (1990), 50:167 $-214$

Cactaceae, Estudios citognéticos de dos especies y una variedad del género Nyctocereus, $48: 75-80$
Cactaceae to Compositae, Vegetation and flora of the Bolson of Cuatro Cienegas region, Coahuila, Mexico: III, 41:127-151

CALDERON DE RZEDOWSKI, GRACIELA. Tigridia martinezii, una especie nueva de iridáceas del estado de Hidalgo (México), 47:3-6

CALDERON DE RZEDOWSKI, GRACIELA y LINCOLN CONSTANCE. Una nueva especie de Tauschia (Umbelliferae) del Estado de México, 41:19-21

CALDERON, M. DEL C., H. CORTEZ, C. PUCHET, M. RAMIREZ, R. VILLALON, E. ZAPATA, LILA LORENZO, A. RAMIREZ ROA, M.A. SOTO ARENAS y A. BRECEDA. Notas sobre la fitogeografía del bosque de montaña en la Sierra del Sur, México. 44:97-102 California, Comparative photosynthetic characteristics of coastal and desert plants of, 42:19-33

CALVERT, WILLIAM H., WILLOW ZUCHOWSKI Y LINCOLN P. BROWER. The impact of forest thinning on microclimate in monarch butterfly (Danaus plexippus L.) overwintering areas of Mexico, 42:1118

CALZADA, JUAN ISMAEL y HUGO PERALES RIVERA. La preservación con alcohol de ejemplares colectados para herbario, 50: $151-155$

CAM en la selva húmeda tropical de .os Tuxtlas, Veracruz, Especies, 45:150153 
Canales resiníferos de la corteza de Bursera copallifera y Bursera grandifolia, Estudio de los, 42:41-54

Caracterización ficoflorística de los paredones de la Sierra de Juárez, Oaxaca. Importancia de las formas de crecimiento algales en la tipificación de un ambiente, 50:121-133

Características palinológicas de las araliáceas de México, 45:117-132

Carbono en plantas superiores, Fotosíntesis y economía del, 49:41-60

CARDENAS S., ANGELES y CLAUDIO DELGADILLO $\mathrm{M}$. Musgos de Zacatecas, México, II, 4613-18; III. Síntesis y fitogeografía, 47:13-24

CARDENAS S., ANGELES y CLAUDIO DELGADILLO $\mathrm{M}$. Musgos de la Península de Yucatán, México. II., 43:35-37

Carpodiptera ameliae (Tiliaceae), en México, Nota sobre la distribución de, 41:166

CARREON ABUD, YASMIN y HECTOR M. HERNANDEZ. Notas sobre la ecología reproductiva de árboles en un bosque mesófilo de montaña en Michoacán, México, 47:25-35

CARVAJAL MORENO, MAGDA. Distribución de Rhynchosporium secalis en el mundo y en México, 48:311

CARVAJAL MORENO, MAGDA. Rango de hospedantes de Rhynchosporium secalis, 46:19-24

Casimiroa (Rutaceae) de la zona árida oaxaqueño-poblana, Nueva especie de, 41:23-26
Casimiroa calderonii Chiang et Medrano. Nota sobre la ortografía correcta de, 46:89

CASTELLANOS V., ALEJANDRO E. Fotosíntesis y economía del carbono en plantas superiores, 49:41-60.

Celastraceae de México, Rzedowskia, un nuevo género de, 41:41-46

Central American Randia (Rubiaceae, Gardenieae), New taxa and a new name in Mexican and, 47:37-48 Cerro Tetzcotzinco, Texcoco, Estado de México, Inventario florístico en el, 48:81-94

CHEHAIBAR NADER, TERE. Reseña de nuevos libros, 43:95-96; 44:104-105

CHEHAIBAR, TERESA y ROSAURA GRETHER. Anatomía de la Madera de algunas especies del género Mimosa (Leguminosae), 50:3-17

CHIANG, FERNANDO. El número cromosómico de Grabowskia geniculata (Solanaceae), 45:141-142

CHIANG, FERNANDO. El número cromosómico de Lycium leiospernum I.M. Johnston (Solanaceae), 48:145146

CHIANG, FERNANDO. Estudios cromosómicos en Lycium (Solanaceae) de Norteamrica, 43:9-23 CHIANG, FERNANDO. Nota sobre la ortografía correcta de Casimiroa calderonii Chiang et Medrano, 46:89

CHIANG, FERNANDO. Nuevas combinaciones y una nueva variedad de Lycium (Solanaceae) de Norteamérica, 43:5-8

CHIANG, FERNANDO. Una nueva especie de Decazyx (Rutaceae) de Tabasco y Veracruz, 43:1-3 
CHIANG, FERNANDO. y FRANCISCO GONZALEZ-MEDRANO. Nueva especie de Casimiroa (Rutaceae) de la zona árida oaxaqueño-poblana, 41:23-26

CHIANG, FERNANDO, JOSE LUIS VILLASEÑOR y PATRICIA DAVILA. Fitogeografía del Valle de Tehuacán-Cuicatlán, 50:135-149

Chiranthodendron pectadactylon Larr. (Sterculiaceae), Nota sobre la propagación y pérdida de viabilidad de las semillas de, 50:157-159

Chloridoideae, Estudio biosistemático de Eragrostis mexicana, E. neomexicana, $E$. orcuttiana y $E$. virescens, Gramineae, 48:95-112

Ciperáceas del Valle de México, Nota sobre tres especies de, 44:95-96

Ciperáceas para la flora del Valle de México y de la República Mexicana, Nuevos registros de, 44:17-21

Citogenética de dos especies y una variedad del género Nyctocereus (Cactaceae), 48:75-80

Clave genérica para las compuestas de la cuenca del río Balsas, 47:65-86

Cnidoscolus spinosus (Euphorbiaceae) en Jalisco, Componentes fenológicos del sistema de cruzamiento monoico de, 42:1-9

Coahuila, Mexico: III Cactaceae to Compositae, Vegetation and flora of the Bolson of Cuatro Cienegas region, 41:127-151

Co-evolución, sobre el significado del "co" en, 44:91-94

Colima, México, Musgos alpinos del Nevado de, 47:7-11
Colubrina johnstonii sp. nov. (Rhamnaceae), Plantae uxpanapae I., 44:8190

Comelináceas, Estudios cromosómicos en seis especies mexicanas de, 50:77 $-87$

Comparative photosynthetic characteristics of coastal and desert plants of California, 42:19-33

Componentes fenológicos del sistema de cruzamiento monoico de Cnidoscolus spinosus (Euphorbiaceae) en Jalisco, 42:1-9

Compositae, Notas taxonómicas y observaciones sobre algunas especies mexicanas de Gnaphalium, 45:15-21

Compositae, Vegetation and flora of the Bolson of Cuatro Cienegas region, Coahuila, Mexico: III Cactaceae to, 41:127-151

Compositae-Astereae del Valle de México, Dos especies nuevas de, 45:111-116

Compositae-Mutisieae, Algunas adiciones al género Acourtia, 45:97109

Compuestas de la cuenca del río Balsas, Clave genérica para las, 47:65-86

Condalia velutina (Rhamnaceae) una planta muy poco colectada, 45:143146

Conservación de especies vegetales, problemas y aplicaciones, El almacenamiento de semillas en la, 49:61-69

Consideraciones sobre la exina en Yucca, observada al microscopio de luz y microscopio de barrido, 48:153-154

CONSTANCE, LINCOLN y GRACIELA CALDERON DE RZFDOWSKI. Una nueva especie de 
Tauschia (Umbelliferae) del Estado de México, 41:19-21

Convolvulaceae en México, Los géneros de, 44:3-16

CORTEZ H., C, PUCHET, M. RAMIREZ R. VILLALON, E. ZAPATA, LILA LORENZO, A. RAMIREZ ROA, M.A. SOTO ARENAS, A. BRECEDA y M. DEL C. CALDERON. Notas sobre la fitogeografía del bosque mesófilo de montaña en la Sierra Madre del Sur, México, 44:97-102

Corteza de Bursera copallifera y Bursera grandifolia, Estudio de los canales resiníferos de la, 42:41-54

Crescentia alata (Bignoniaceae), Parasitismo floral por abejas sociales (Meliponinae; Apidae) en el árbol quiropterófilo, 50:69-76

Cromosomas en seis especies mexicanas de comelináceas, 50:77-87

Cruzamiento monoico de Cnidoscolus spinosus (Euphorbiaceae) en Jalisco, Componentes fenológicos del sistema de, 42:1-9

Cuatro Cienegas region, Coahuila, Mexico: III Cactaceae to Compositae, Vegetation and flora of the Bolson of, 41:127-151

Cuenca del río Balsas, Clave genérica para las compuestas de la, 47:65-86 Cyperaceae en el Valle de México, 44:17-21, 95-96; 45:39-47; 46:29-35

Cyperaceae en el Valle de México. I. Afinidades ecológicas, Algunos aspectos ecológicos y fitogeográficos de las especies de, 45:39-47; II. Análisis fitogeográfico, 46:29-35

Cyperaceae nuevos registros, 44:17-21
Danaus plexippus L. overwintering areas of Mexico, The impact of forest thinning on microclimate in monarch butterfly, 42:11-18

Decazyx (Rutaceae) de Tabasco y Veracruz, Una nueva especie de, 43: 1-3

DELGADILLO M. CLAUDIO. Musgos alpinos del Nevado de Colima, México, 47:7-11

DELGADILLO M., CLAUDIO y ANGELES CARDENAS S. Musgos de Zacatecas, México, II, 46:13-18; III. Síntesis y fitogeografía, 47:13-24

DELGADILLO M., CLAUDIO y ANGELES CARDENAS S. Musgos de la Península de Yucatán, México. II., 43:35-37

DELGADILLO M., CLAUDIO y SERGIO ZAMUDIO. Algunos musgos de Tabasco, México, 48:13-18

DELGADO SALINAS, ALFONSO, ENRIQUE MARTINEZ HERNANDEZ y PILAR FERNANDEZ ORTUÑO. Estudio del polen de Phaseolus chiapasanus Piper (Leguminosae: Phaseolinae), 43:2534

Demografía y crecimiento del lirio acuático (Eichhornia crassipes), 45:71-83 Desert plants of California, Comparative photosynthetic characteristics of, 42:19-33

Detalles ultraestructurales de la madera de algunas Boraginaceae de México, 45:3-14

Digitaria decumbens, Productividad primaria de tres praderas de especies tropicales: pará (Brachiaria mutica), grama amarga (Paspalum conjugatum) y pangola, 41:3-18 
DIRZO, RODOLFO. Sobre el significado del "co" en co-evolución, 44:91-94

DIRZO, RODOLFO. ADRIAN FERNANDEZ BREMAUNTZ, GABRIELA GOMEZ RODRIGUEZ y VERONICA GONZALEZ KLADIANO. Un aparato sencillo para medir la dureza del follaje en estudios de herbivoría, 43:81-88

Distribución cosmopolita, Sobre la ecología reproductiva de Nicotiana glauca Grah: una maleza de, 41:47-73

Distribución esporangial en estróbilos de especies de Selaginella, 41:33-40

Distribución de Carpodiptera ameliae (Tiliaceae), en México, Nota sobre la, 41:166

Distribución de Rhynchosporium secalis en el mundo y en México, 48:3-11 Distribución de Savia sessiliflora (Euphorbiaceae) en México, Nota sobre la, 47:90-91

Distribution of the genus Pinus in the Western United States and Mexico, The altitudinal, 42:55-71

Dos especies nuevas de CompositaeAstereae del Valle de México, 45:111116

Dos nuevas especies mexicanas de la familia Rubiaceae, 44:73-80

DRECKMANN KURT M., ABEL SENTIES G. y FRANCISCO F. PEDROCHE. La familia Rhodomelaceae (Ceramiales, Rhodophyta en la costa del estado de Michoacán, México, 50:89-120

DRECKMANN, KURT M., FRANCISCO F. PEDROCHE y ABEL SENTIES G. Lista florística de las algas marinas bentónicas de la costa norte de Michoacán, México, 50:19-42

DWYER, JOHN D. y DAVID $H$. LORENCE. New taxa and a new name in Mexican and Central American Randia (Rubiaceae, Gardenieae), 47:37-48

DWYER, JOHN D. y DAVID H. LORENCE. New taxa in Mexican Psychotria (Rubiaceae, Psychotrieae), 47:49-64

DAVILA, PATRICIA, FERNANDO CHIANG y JOSE LUIS VILLASEÑOR. Fitogeografía del Valle de Tehuacán-Cuicatlán, 50:135.

Ecofisiología de las micorrizas, 49:1930.

Ecofisiología vegetal, El papel del microclima en la, 49:31-39. Ecología del fitocromo: su función en las semillas, Fisiología y, 49:71-84.

Ecología reproductiva de Nicotiana glauca Grah: una maleza de distribución cosmopolita, Sobre la, 41:47-73

Ecología reproductiva de árboles en un bosque mesófilo de montaña en Michoacán, México, Notas sobre la, 47:25-35

Ecología y fisiología de plantas, 49:3-5 Ecología y fitogeografía de Cyperaceae, 45:39-47; 46:29-35

EGUIARTE, LUIS E. y ALBERTO BURQUEZ. Reducción en la fecundidad en Manfreda brachystachya (Cav.) Rose, una agavácea polinizada por murciélagos: los riesgos de la especialización en la polinización. 48:147-149

EHLERINGER, J., H. A. MOONEY, J. BERRY y O. BJORKMAN. COM- 
parative photosyntheticcharacteristics of coastal and desert plants of California, 42:19-33

Eichhornia crassipes (Mart) Solms: dinámica de crecimiento en dos localidades selectas de México, Estudio demográfico del lirio acuático, 45:71-83

Elaeagia (Rubiaceae) in Mexico, with description of a new species, $E$. $u x-$ panapensis, First record of 45:65-69

Elaeagia uxpanapensis, First record of Elaeagia (Rubiaceae) in Mexico, with description of a new species, 45:65-69 ENGLEMAN, E. MARK y GUADALUPE SUAREZ RAMOS. Estudio de los canales resiníferos de la corteza de Bursera copallifera y Bursera grandifolia, 42:41-54

Eragrostis mexicana, E. neomexicana $E$. orcuttiana y E. virescens (Gramineae: Chloridoideae), Estudio biosistemático de, 48:95-112

Esclarecimiento taxonómico de $\mathrm{Nym}$ phaea gracilis Zucc., planta acuática endémica de México, 45:85-95

Especie nueva de Stegnosperma (Phytolaccaceae) del suroeste de Puebla, México, Una, 46:37-41

Especies CAM en la selva húmeda tropical de los Tuxtlas, Veracruz, 45:150153

ESPINOSA GARCIA, FRANCISCO JAVIER. Adiciones a la flora arvense del Valle de México, 41:27-32.

ESPINOSA GARCIA, FRANCISCO JAVIER. Nota sobre la identidad del segundo Tzonpotónic mencionado por Francisco Hernández en su Historia Natural de la Nueva España, 47:87-89
ESPINOSA GARCIA, FRANCISCO JAVIER. Notas taxonómicas y observaciones sobre algunas especies mexicanas de Gnaphalium (Compositae), 45:15-21

ESPINOSA GARCIA, FRANCISCO

JAVIER. Reseña de libros, 46:93

Esporangios en estróbilos de Selaginella, 41:33-40

Esporas de helechos en el suelo de Los Tuxtlas, Ver. El banco de, 43:89-92 Estado de México, Una nueva especie de Tauschia (Umbelliferae) del, 41:19-21

Estróbilos de especies de Selaginella, Distribución esporangial en, 41:33-40 Estructura y composición la vegetación herbácea de un bosque uniespecífico de Pinus hartwegii I. Estructura y composición florística, 41:75-125

Estudio biosistemático de Eragrostis mexicana, E. neomexicana, E. orcuttiana y E. virescens (Gramineae: Chloridoideae), 48:95-112

Estudio de los canales resiníferos de la corteza de Bursera copallifera y Bursera grandifolia, 42:41-54

Estudio del polen de Phaseolus chiapasanus Piper (Leguminosae: Phaseolinae), 43:25-34

Estudio demográfico del lirio acuático Eichhornia crassipes (Mart) Solms: dinámica de crecimiento en dos localidades selectas de México, 45:7183

Estudios citogenéticos de dos especies y una variedad del género Nyctocereus (Cactaceae), 48:75-80

Estudios cromosómicos de Lycium (Solanaceae) de Norteamérica, 43:923 
Estudios cromosómicos en seis especies mexicanas de comelináceas, 50:77-87

Estudios quimiotaxonómicos de Bursera (Burseraceae) I. Bursera chemapodicta sp. n., 43:73-80

Euphorbiaceae en Jalisco, Componentes fenológicos del sistema de cruzamiento monoico de Cnidoscolus spinosus, 42:1-9

Euphorbiaceae en México, Nota sobre la distribución de Savia sessiliflora (Euphorbiaceae) en, 47:90-91

Euphorbiaceae, nueva especie de la costa de Jalisco, México, Jatropha chamelensis, 42:35-39

Exina en Yucca, observada al microscopio de luz y microscopio de barrido, Consideraciones sobre la, 48:153-154

Familia Rhodomelaceae (Ceramiales, Rhodophyta) en la costa del estado de Michoacán, México, La, 50: 89-120

Familia Rubiaceae en la región de Los Tuxtlas, Veracruz, México, La, 50:43$-68$

Fecundidad en Manfreda brachystachya (Cav.) Rose, una agavácea polinizada por murciélagos: los riesgos de la especialización en la polinización, Reducción en la, 48:147-149

FERNANDEZ BREMAUNTZ, ADRIAN, RODOLFO DIRZO, GABRIELA GOMEZ RODRIGUEZ y VERONICA GONZALEZ KLADIANO. Un aparato sencillo para medir la dureza del follaje en estudios de herbivoría, 43:81-88

FERNANDEZ NAVA, RAFAEL. Condalia velutina (Rhamnaceae) una planta muy poco colectada, 45:143146
FERNANDEZ ORTUÑO, PILAR, ALFONSO DELGADO SALINAS y ENRIQUE MARTINEZ HERNANDEZ. Estudio del polen de Phaseolus chiapasanus Piper (Leguminosae: Phaseolinae), 43:2534

Ficoflorística de los paredones de la Sierra de Juárez, Oaxaca, Caracterización, 50:121-133

First record of Elaeagia (Rubiaceae) in Mexico, with description of a new species, E. uxpanapensis, 45:65-69

Fisiología ecológica de plantas, 49:3-5

Fisiología ecológica vegetal, El papel del microclima en la, 49:31-39

Fisiología y ecología del fitocromo, su función en las semillas, 49:71-84

Fitocromo, su función en las semillas, Fisiología y ecología del, 49:71--84

Fitófagos, Spigelia longiflora (Mart. et Gal.): actividad antialimentaria en tres especies de insectos, 48:135-138 Fitogeografía, Musgos de Zacatecas, México III. Síntesis y, 47:13-24

Fitogeografía del bosque mesófilo de montaña en la Sierra Madre del Sur, México, Notas sobre la, 44:97-102

Fitogeografía del bosque mésofilo de montaña de Teocelo, Veracruz, 48:3563

Fitogeografía del Valle de TehuacánCuicatlán, 50: 135-149

Flora arvense del Valle de México, Adiciones a la, 41:27-32

Flora de México, Métodos computarizados y algunas de sus aplicaciones al estudio de la, 48:65-73 Flora del Valle de México y de la República Mexicana, Nuevos 
registros de ciperáceas para la, 44:1721

Flora of the Bolson of Cuatro Cienegas region, Coahuila, Mexico: III Cactaceae to Compositae, Vegetation and, 41:127-151

FLORES GUIDO, JOSE SALVADOR. Vegetación insular de la Península de Yucatán, 45:23-37

Follaje en estudios de herbivoría, Un aparato sencillo para medir la dureza del, 43:81-88

Fotosíntesis y economía del carbono en plantas superiores, 49:41-60

FRAILE, MA. EUGENIA y RAMON RIBA. Distribución esporangial en estróbilos de especies de Selaginella, 41:33-40

FRYXELL, PAUL A., ANTONIO KRAPOVICKAS y DAVID $M$. BATES. Allosidastrum, un nuevo género de Malvaceae de los neotrópicos, 48:23-34

GALVAN VILLANUEVA, RAQUEL. Nuevos registros de Juncaceae y Liliaceae en el Valle de México, 46:2527

GARCIA FRANCO, JOSE GUADALUPE y HUGO R. PERALES RIVERA. Nota sobre la propagación y pérdida de viabilidd de las semillas de Chiranthodendron pectadactylon Larr. (Sterculiaceae), 50:157 $-159$

GARCIA MOYA, EDMUNDO y J. JESUS TELLO BALDERAS. El maguey (Agave, subgénero Agave) en el altiplano potosino-zacatecano, 48:119-134
Gardenieae, New taxa and new name in Mexican and Central American Randia Rubiaceae, 47:37-48

Género nuevo de la parte meridional de México: Gypsacanthus (Acanthaceae, Justicieae, Odontoneminae), Un, 46:47-51

Géneros de Convolvulaceae en México, Los, 44:3-16

Gleditsia (Leguminosae), Nuevo registro para México de, 48:143-144

Gnaphalium (Compositae), Notas taxonómicas y observaciones sobre algunas especies mexicanas de, 45:15-21 GOMEZ RODRIGUEZ, GABRIELA, RODOLFO DIRZO, ADRIAN FERNANDEZ BREMAUNTZ y VERONICA GONZALEZ KLADIANO. Un aparato sencillo para medir la dureza del follaje en estudios de herbivoría, 43:81-88

GONZALEZ E., MA. SOCORRO. Nota sobre tres especies de ciperáceas del Valle de México, 44:95-96

GONZALEZ E., MA. SOCORRO. Nuevos registros de ciperáceas para la flora del Valle de México y de la República Mexicana, 44:17-21

GONZALEZ E., SOCORRO y JERZY RZEDOWSKI. Algunos aspectos ecológicos y fitogeográficos de las especies de Cyperaceae en el Valle de México. I. Afinidades ecológicas, 45:39-47; II. Análisis fitogegráfico, 46:29-35.

GONZALEZ GONZALEZ, JORGE y ROSA LUZ TAVERA SIERRA. Caracterización ficoflorística de los paredones de la Sierra de Juárez, Oaxaca, México. Importancia de las 
formas de crecimiento algales en la tipificación de un ambiente, 50:121 GONZALEZ KLADIANO, VERONICA, RODOLFO DIRZO, ADRIAN FERNANDEZ BREMAUNTZ, GABRIELA GOMEZ RODRIGUEZ. Un aparato sencillo para medir la dureza del follaje en estudios de herbivoría, 43:81-88

GONZALEZ-MEDRANO, FRANCISCO Mimosa martindelcampoi Medrano(Leguminosae), una especie nueva del sudoeste de Tamaulipas, México, 43:39-42

GONZALEZ-MEDRANO, FRANCISCO Rzedowskia, un nuevo género de Celastraceae de México, 41:41-46 GONZALEZ-MEDRANO, FRANCISCO y FERNANDO CHIANG. Nueva especie de Casimiroa (Rutaceae) de la zona árida oaxaqueño-poblana, 41:23-26

GONZALEZ-MEDRANO, FRANCISCO y ROSALINDA MEDINA LEMOS. Una especie nueva de Stegnosperma (Phytolaccaceae) del suroeste de Puebla, México, 46:37-41 GONZALEZ-MEDRANO, FRANCISCO y VICTOR JARAMILLO LUQUE. Análisis de la vegetación arbórea en la provincia florística de Tehuacán-Cuicatlán, 45:49-64

GONZALEZ-MEDRANO, FRANCISCO, T.P. RAMAMOORTHY y P. HIRIART VALENCIA. Neoeplingia Ramamoorthy, Hiriart Medrano (Labiatae) un nuevo género de Hidalgo, México, 43:61-65

Grabowskia geniculata (Solanaceae), El número cromosómico de, 45:141-142
Grama amarga (Paspalum conjugatum) y pangola (Digitaria decumbens), Productividad primaria de tres praderas de especies tropicales: para (Brachiaria mutica), 41:3-18

Gramineae Chloridoideae, Estudio biosistemático de Eragrostis mexicana, E. neomexicana, E. orcuttiana y $E$. virescens, 48:95-112

Gramineae, Registros nuevos de plantas acuáticas mexicanas. II Luziola subintegra Swallen y L. spruceana Benth. ex Doell, 46:90-91

Gramíneas del Valle de México, Nuevos registros y nuevas combinaciones de, 48:19-22

GRETHER, ROSAURA. Aspectos ecológicos de Mimosa biuncifera y Minosa monancistra en el noroeste del estado de Guanajuato, 43:43-60

GRETHER, ROSAURA. Nota sobre la identidad del tepescohuite en México, 48:151-152

GRETHER, ROSAURA y TERESA CHEHAIBAR. Anatomía de la madera de Algunas especies del género Mimosa (Leguminosae), 50:3-17

Guanajuato, Aspectos ecológicos de Mimosa biuncifera y Mimosa monancistra en el noroeste del estado de, 43:43-60

Guerrero, México, Selaginella rzedowskii, Una nueva especie de selaginela heterófila del estado de, 44:23-27

Gypsacanthus (Acanthaceae, Justicieae, Odontoneminae), Un género nuevo de la parte meridional de México:, $46: 47-51$ 
Haloragaceae, Registros nuevos de plantas acuáticas mexicanas I: Myriophyllum quitense H.B.K. 45:147-149

Helechos de Veracruz: Adiantum (Pteridaceae), 44:43-62

Helechos en el suelo de Los Tuxtlas, Ver., El banco de esporas de, 43:89-92 Herbario, La preservación con alcohol de ejemplares colectados para, 50:151 $-155$

Herbivoría, Un aparato sencillo para medir la dureza del follaje en estudios de, 43:81-88

Hernández, Francisco en su Historia Natural de la Nueva España, Nota sobre la identidad del segundo Tzonpotónic mencionado por, 47:8789

HERNANDEZ, HECTOR MANUEL Sobre la ecología reproductiva de Nicotiana glauca Grah: una maleza de distribución cosmopolita, 41:47-73

HERNANDEZ, HECTOR MANUEL y YASMIN CARREON ABUD. Notas sobre la ecología reproductiva de árboles en un bosque mesófilo de montaña en Michoacán, México, 47:25-35

HERRERA A., YOLANDA. Nuevos registros y nuevas combinaciones de gramíneas del Valle de México, 48:1922

Hidalgo, México, Neoeplingia Ramamoorthy, Hiriart Medrano (Labiatae) un nuevo género de, 43:6165

Hidalgo, México, Tigridia martinezii, una especie nueva de iridáceas del estado de, 47:3-6

Hidalgo, México, Una nueva especie de Scutellaria (Labiatae) de, 46:43-46
HIRIART VALENCIA, PATRICIA. Una nueva especie de Scutellaria (Labiatae) de Hidalgo, México, 46:4346

HIRIART VALENCIA, PATRICIA, T.P. RAMAMOORTHY y FRANCISCO GONZALEZ-MEDRANO. Neoeplingia Ramamoorthy, Hiriart Medrano (Labiatae) un nuevo género de Hidalgo, México, 43:61-65

Historia Natural de la Nueva España, Nota sobre la identidad del segundo Tzonpotónic mencionado por Francisco Hernández en su, 47:87-89

Hospedantes de Rhynchosporium secalis, Rango de, 46:19-24

HUANTE, PILAR y EMANUEL RINCON. Nutrición mineral, 49:7-17

IBARRA MANRIQUEZ GUILLERMO y DAVID H. LORENCE. La familia Rubiaceae en la región de Los Tuxtlas, Veracruz, México, 50:00-00. Identidad del segundo Tzonpotónic mencionado por Francisco Hernández en su Historia Natural de la Nueva España, Nota sobre la, 47:8789

Impact of forest thinning on microclimate in monarch butterfly (Danaus plexippus L.) overwintering areas of Mexico, The, 42:11-18

Indice acumulativo del Boletín de la Sociedad Botánica de México de los números 41 (1981) al 50 (1990), 50:167 $-214$

Insectos fitófagos, Spigelia longiflora (Mart. et. Gal.): actividad antialimentaria en tres especies de, 48:135-138 Interacción entre radiaciones gamma y mms sobre la plántula de semillas de. 
Triticale pretratadas con ácido ascór. bico, Análisis de la, 44:63-71

Interpretaciones sobre la lluvia de polen en la región de San Luis Potosí, México, 46:53-74

Inventario florístico en el cerro Tetzcotzinco, Texcoco, Estado de México, 48:81-94

Iridáceas del estado de Hidalgo (México), Tigridia martinezii, una especie nueva de, 47:3-6

Jalisco, Componentes fenológicos del sistema de cruzamiento monoico de Cnidoscolus spinosus (Euphorbiaceae) en, 42:1-9

Jalisco, México, Jatropha chamelensis (Euphorbiaceae), nueva especie de la costa de, 42:35-39

JARAMILLO LUQUE, VICTOR y FRANCISCO GONZALEZ- MEDRANO. Análisis de la vegetación arbórea en la provincia florística de Tehuacán-Cuicatlán, 45:49-64

JARAMILLO LUQUE, VICTOR, EMILY J. LOTT y JERZY RZEDOWSKI. Un género nuevo de la parte meridional de México: Gypsacanthus (Acanthaceae, Justicieae, Odontoneminae), 46:47-51

Jatropha chamelensis (Euphorbiaceae), nueva especie de la costa de Jalisco, México, 42:35-39

Juncaceae y Liliaceae en el Valle de México, Nuevos registros de, 46:25-27 KOCH, STEPHEN D. e ISIDORO SANCHEZ VEGA. Estudio biosistemático de Eragrostis mexicana, E. neomexicana, E. orcuttiana y $E$. virescens (Gramineae: Chloridoideae), 48:95-112
KOCH, STEPHENM. y MA. TERESA P.PULIDO.Inventario florístico en el cerro Tezcotzinco, Texcoco, Estado de México, 48:81-94

KRAPOVICKAS, ANTONIO, PAUL A. FRYXELL y DAVID M. BATES. Allosidastrum, un nuevo género de Malvaceae de los neotrópicos, 48:2334

Labiatae de Hidalgo, México. Una nueva especie de Scutellaria, 46:43-46

Labiatae, un nuevo género de Hidalgo, México, Neoeplingia Ramamoorthy, Hiriart Medrano, 43:61-65

Lagos-cráter del estado de Puebla, México, La vegetación acuática vascular de seis, 46:75-88

Lectotipificación de Limnocharis laforestii Duchass. ex Griseb. (Limnocharitaceae), 47:92-93

Leguminosae, Anatomía de la madera de algunas especies del género Mimosa, 50:3-17

Leguminosae, Dos nuevas especies para México, Acacia velvae y $A$. Mirandae, 43: $67-71$

Leguminosae, Nuevo registro para Mexico de Gleditsia, 48:143-144

Leguminosae, Phaseolinae, Estudio del polen de Phaseolus chiapasanus Piper, 43:25-34

Leguminosae, Una especie nueva del sudoeste de Tamaulipas, México, Mimosa martindelcampoi Medrano, 43:39-42

Leguminosae en México: O. panamensis, Una adición al género Ormosia, 48:155-158

Leguminosae in Mexico, including a new species from Oaxaca, Ormosia, 41:153-159 
Liliaceae en el Valle de México, Nuevos registros de Juncaceae y, 46:25-27

Limnocharis laforestii Duchass. ex Griseb. (Limnocharitaceae), Lectotipificación de, 47:92-93

Limnocharitaceae, Lectotipificación de Limnocharis laforestii Duchass. ex Griseb, 47:92-93

Lirio acuático Eichhornia crassipes (Mart) Solms: dinámica de crecimiento en dos localidades selectas de México, Estudio demográfico del, 45:71-83

Lista florística de las algas marinas bentónicas de la costa norte de Michoacán, México, 50:19-42

Lluvia de polen en la región de San Luis Potosí, México, Interpretaciones sobre la, 46:53-74

LOREA-HERNANDEZ, FRANCISCO G. Selaginella rzedowskii, una nueva especie de selaginela heterófila del estado de Guerrero, México, 44:23-27

LORENCE, DAVID H. First record of Elaeagia (Rubiaceae) in Mexico, with description of a new species, $E$. uxpanapensis, 45:65-69

LORENCE, DAVID H. y GUILLERMO IBARRA MANRIQUEZ. La familia Rubiaceae en la región de Los Tuxtlas, Veracruz, México, 50:43-68

LORENCE, DAVID H. y JOHN D. DWYER. New taxa and a new name in Mexican and Central American Randia (Rubiaceae, Gardenieae), 47:37-48

LORENCE, DAVID H. y JOHN D. DWYER. New taxa in Mexican Psychotria (Rubiaceae, Psychotrieae), 47:49-64
LORENZO, LILA, A. RAMIREZ ROA, M.A. SOTO ARENAS, A. BRECEDA, M. DEL C. CALDERON, H. CORTEZ, C. PUCHET, M. RAMIREZ, R. VILLALON y E. ZAPATA. Notas sobre la fitogeografía del bosque mesófilo de montaña en la Sierra Madre de Sur, México, 44:97-102

LORENZO, LILA, ISOLDA LUNA, LUCIA ALMEIDA y LOURDES VILLERS. Reconocimiento florístico y consideraciones fitogeográficas del bosque mesófilo de montaña de Teocelo, Veracruz, 48:35-63

Los Tuxtlas, Veracruz, México, La familia Rubiaceae en la región de, 50:43-68

LOT HELGUERAS, ANTONIO y ALEJANDRO NOVELO RETANA. Esclarecimiento taxonómico de Nymphaea gracilis Zucc., planta acuática endémica de México, 45:8595

LOT HELGUERAS, ANTONIO y MARTHA S. NIÑO SULKOWSKA. Estudio demográfico del lirio acuático Eichhornia crassipes (Mart) Solms: dinámica de crecimiento en dos localidades selectas de México, 45:71-83

LOTT, EMILY J. Nota sobre la distribución de Savia sessiliflora (Euphorbiacae) en México, 47:90-91

LOTT, EMILY J., VICTOR JARAMILLO L. y JERZY RZEDOWSKI. Un género nuevo de la parte meridional de México: Gypsacanthus (Acanthaceae, Justicieae, Odontoneminae), 46:47-51 
LOZANO-GARCIA, SOCORRO. Interpretaciones sobre la lluvia de polen en la región de San Luis Potosí, México, 46:53-74

LUDLOW-WIECHERS, BEATRIZ y LINA OJEDA. El pólen del género Agave para la Península de Yucatán, 44:29-42

LUDLOW-WIECHERS, BEATRIZ, LINA OJEDA Y RODOLFO PALACIOS-CHAVEZ. Consideraciones sobre la exina en $Y u c c a$, observada al microscopio de luz y microscopio de barrido, 48:153-154

LUNA, ISOLDA, LUCIA ALMEIDA, LOURDES VILLERS y LILA LORENZO. Reconocimiento florístico y consideraciones fitogeográficas del bosque mesófilo de montaña de Teocelo, Veracruz, 48:35-63

Luziola subintegra Swallen y L. spruceana Benth. ex Doell. (Gramineae), Registros nuevos de plantas acuáticas mexicanas. II, 46:90-91

Lycium (Solanaceae) de Norteamérica, Estudios cromosómicos en, 43:9-23

Lycium (Solanaceae) de Norteamérica, Nuevas combinaciones y una nueva variedad de, 43:5-8

Lycium leiospermum I.M. Johnston (Solanaceae), El número cromosómico de, 48:145-146

Madera de algunas Boraginaceae de México, Detalles ultraestructurales de la, 45:3-14

Madera de algunas especies del género Mimosa (Leguminosae), Anatomía de la, 50:3-17
Maguey (Agave, subgénero Agave) en el altiplano potosino-zacatecano, El, 48:119-134

Maleza de distribución cosmopolita, Sobre la ecología reproductiva de Nicotiana glauca Grah: una, 41:47-73

Malvaceae de los neotrópicos, Allosidastrum, un nuevo género de, 48:23-34 Manfreda brachystachya (Cav.) Rose, una agavácea polinizada por murciélagos: los riesgos de la especialización en la polinización, Reducción en la fecundidad en, 48:147-149

MARTINEZ ALMERAYA, PATRICIA, PEDRO MERCADO RUARO, GUADALUPE PALOMINO HASBACH y BALBINA VAZQUEZ BENITEZ. Estudios cromosómicos en seis especies mexicanas de comelináceas, 50:77-87

MARTINEZ DEL RIO, CARLOS y STEPHEN H. BULLOCK. Parasitismo floral por abejas sociales (Meliponinae; Apidae) en el árbol quiropterófilo Crescentia alata (Bignoniaceae), 50:69-76

MARTINEZ HERNANDEZ, ENRIQUE, ALFONSO DELGADO SALINAS y PILAR FERNANDEZ ORTUÑO. Estudio del polen de Phaseolus chiapasanus Piper (Leguminosae: Phaseolinae), 43:2534

MEDINA LEMOS, ROSALINDA y FRANCISCO GONZALEZMEDRANO. Una especie nueva de Stegnosperma (Phytolaccaceae) del suroeste de Puebla, México, 46:37-41 
MENDOZA, ANA, ERNST-DETLEF SCHULZE, HAROLD A. MOONEY Y STEPHEN $H$. BULLOCK. Water contents of wood of tropical deciduous forest species during the dry season, 48:113-118

MERCADO RUARO, PEDRO, GUADALUPE PALOMINO HASBACH, BALBINA VAZQUEZ BENITEZ y PATRICIA MARTINEZ ALMERAYA. Estudios cromosómicos en seis especies mexicanas de comelináceas, 50:77-87

Métodos computarizados y algunas de sus aplicaciones al estudio de la flora de México, 48:65-73

Mexican and Central American Randia (Rubiaceae, Gardenieae), New taxa and a new name in, 47:37-48

Mexican Psychotria (Rubiaceae, Psychotrieae), New taxa in, 47:49-64

México, Características palinológicas de las araliáceas de, 45:117-132

México, Detalles ultraestructurales de la madera de algunas Boraginaceae de, 45:3-14

México, Distribución de Rhynchosporium secalis en el mundo y en, 48:3-11

México, Esclarecimiento taxonómico de Nymphaea gracilis Zucc., planta acuática endémica de, 45:85-95

México, Gypsacanthus (Acanthaceae, Justicieae, Odontoneminae), Un género nuevo de la parte meridional de, 46:47-51

México, Los géneros de Convolvulaceae en, 44:3-16

México, Nota sobre la distribución de Carpodiptera ameliae (Tiliaceae), en, 41:166
México, Nota sobre la distribución de Savia sessiliflora (Euphorbiaceae) en, 47:90-91

México, Nota sobre la identidad del tepescohuite en, 48:151-152

México, Rhizophora harrisonii (Rhizophoraceae), un nuevo registro para las costas de, 41:163-165

México, Rzedowskia, un nuevo género de Celastraceae de, 41:41-46

Mexico, The altitudinal distribution of the genus Pinus in the Western United States and, 42:55-71

Mexico, The impact of forest thinning on microclimate in monarch butterfly (Danaus plexippus L.) overwintering areas of, 42:11-18

Michoacán, México, La familia Rhodomelaceae (Ceramiales, Rhodophyta) en la costa del estado de, 50:89-120

Michoacán, México, Lista florística de las algas marinass bentónicas de la costa norte de, 50:19-42

Michoacán, México, Notas sobre la ecología reproductiva de árboles en un bosque mesófilo de montaña en, 47:25-35

Micorrizas, Aspectos ecofisiológicos de las, 49:19-30

Microclima en la fisiología ecológica vegetal, El papel del, 49:31-39

Microclimate in monarch butterfly (Danaus plexippus L.) overwintering areas of Mexico, The impact of forest thinning on, 42:11-18

Microscopio de luz y microscopio de barrido, Consideraciones sobre la exina en Yucca, observada al, 48:153-154 
Mimosa (Leguminosae), Anatomía de la madera de algunas especies del género, 50:3-17

Mimosa biuncifera y Mimosa monancistra en el noroeste del estado de Guanajuato, Aspectos ecológicos de, 43:43-60

Mimosa martindelcampoi Medrano (Leguminosae), una especie nueva del sudoeste de Tamaulipas, México, 43:39-42

Mimosa monancistra en el noroeste del estado de Guanajuato, Aspectos ecológicos de Mimosa biuncifera y, 43:43-60

Monarch butterfly (Danaus plexippus L.) overwintering areas of Mexico, Impact of forest thinning on microclimate in, 42:11-18

MOONEY, H. A., J. BERRY, O. BJORKMAN y J. EHLERINGER. Comparative photosynthetic characteristics of coastal and desert plants of California, 42:19-33

MOONEY, HAROLD A., ERNSTDETLEF SCHULZE, STEPHEN H. BULLOCK, y ANA MENDOZA. Water contents of wood of tropical deciduous forest species during the dry season, 48:113-118

MORENO, NANCY P. y ROBERT ALLKIN. Métodos computarizados y algunas de sus aplicaciones al estudio de la flora de México, 48:65-73

Musgos alpinos del Nevado de Colima, México, 47:7-11

Musgos de Tabasco México, Algunos, 48:13-18

Musgos de Zacatecas, México. II, 46:1318; III. Síntesis y fitogeografía, 47:1324.
Musgos de la Península de Yucatán, México. II., 43:35-37

Mutisieae, Algunas adiciones al género Acourtía Compositae, 45:97-109

Myriophyllum quitense H.B.K. (Haloragaceae). Registros nuevos de plantas acuáticas mexicanas I: 45:147149

Neoeplingia Ramamoorthy, Hiriart Medrano (Labiatae) un nuevo género de Hidalgo, México, 43:61-65

Neotrópicos, Allosidastrum, un nuevo género de Malvaceae de los, 48:23-34 New taxa and a new name in Mexican and Central American Randia (Rubiaceae, Gardenieae), 47:37-48 New taxa in Mexican Psychotria (Rubiaceae, Psychotrieae), 47:49-64 Nicotiana glauca Grah: una maleza de distribución cosmopolita, Sobre la ecología reproductiva de, 41:47-73

NIÑO SULKOWSKA, MARTHA S. y ANTONIO LOT HELGUERAS. Estudio demográfico del lirio acuático Eichhomia crassipes (Mart) Solms: dinámica de crecimiento en dos localidades selectas de México, 45:7183

Norteamérica, Estudios cromosómicos en Lycium (Solanaceae) de, 43:9-23

Norteamérica, Nuevas combinaciones y una nueva variedad de Lycium (Solanaceae) de, 43:5-8

Nota aclaratoria sobre las hojas de Valeriana vaginata (Valerianaceae), 41:167-170

Nota sobre la distribución de Carpodiptera ameliae (Tiliaceae), en México, 41:166 
Nota sobre la distribución de Savia sessiliflora (Euphorbiaceae) en México, 47:90-91

Nota sobre la identidad del segundo Tzonpotónic mencionado por Francisco Hernández en su Historia Natural de la Nueva España, 47:87-89

Nota sobre la identidad del tepescohuite en México, 48:151-152

Nota sobre la ortografía correcta de Casimiroa calderonii Chiang et Medrano, 46:89

Nota sobre la propagación y pérdida de viabilidad de las semillas de Chiranthodendron pectadactylon Larr. (Sterculiaceae), 50:157-159

Nota sobre tres especies de ciperáceas del Valle de México, 44:95-96

Notas botánicas, 41:161-170; 43:81-96; 44:91-102; 45:141-153; 46:89-91; 47:87-93; 48:139-158; 50:157-159

Notas sobre la ecología reproductiva de árboles en un bosque mesófilo de montaña en Michoacán, México, 47:25-35

Notas sobre la fitogeografía del bosque mesófilo de montaña en la Sierra Madre del Sur, México, 44:97-102

Notas sobre las perturbaciones naturales en un bosque mesófilo de montaña en Tamaulipas, 48:139-142

Notas taxonómicas y observaciones sobre algunas especies mexicanas de Gnaphalium (Compositae), 45:15-21

NOVELO RETANA, ALEJANDRO Lectotipificación de Limnocharis laforestii Duchass. ex Griseb. (Limnocharitaceae), 47:92-93

NOVELO RETANA, ALEJANDRO Nuevo registro para México de Pontederia rotundifolia, 41:163-165
NOVELO RETANA, ALEJANDRO Registros nuevos de plantas acuáticas mexicanas I: Myriophyllum quitense H.B.K.

(Haloragaceae), 45:147-149; II Luziola subintegra Swalen y L. spruceana Benth ex Doell. (Gramineae), 46:9091

NOVELO RETANA, ALEJANDRO y ANTONIO LOT HELGUERAS. Esclarecimiento taxonómico de $\mathrm{Nym}$ phaea gracilis Zucc., planta acuática endémica de México, 45:85-95

NOVELO RETANA, ALEJANDRO y PEDRO RAMIREZ-GARCIA. La vegetación acuática vascular de seis lagos-cráter del estado de Puebla, México, 46:75-88

Nueva especie de Casimiroa (Rutaceae) de la zona árida oaxaqueño-poblana, 41:23-26

Nueva especie de Decazyx (Rutaceae) de Tabasco y Veracruz, Una, 43:1-3

Nueva especie de Scutellaria (Labiatae) de Hidalgo, México, Una, 46:43-46

Nueva especie de Tauschia (Umbelliferae) del Estado de México, Una, 41:19-21

Nuevas combinaciones y una nueva variedad de Lycium (Solanaceae) de Norteamérica, 43:5-8

Nuevo registro para México de Gleditsia (Leguminosae), 48:143-144

Nuevo registro para México de Pontederia rotundifolia, 41:161

Nuevos registros de ciperáceas para la flora del Valle de México y de la República Mexicana, 44:17-21

Nuevos registros de Juncaceae y Liliaceae en el Valle de México. 46:2527 
Nuevos registros y nuevas combinaciones de gramíneas del Valle de México, 48:19-22

Número cromosómico de Grabowskia geniculata (Solanaceae), El, 45:141142

Número cromosómico de Lycium leiospermum I.M. Johnston (Solanaceae), El, 48:145-146

Nutrición mineral, 49:7-17 Nyctocereus (Cactaceae), Estudios citogenéticos de dos especies y una variedad del género, 48:75-80

Nymphaea gracilis Zucc., planta acuática endémica de México, Esclarecimiento taxonómico de, 45:85-95

Oaxaca, Importancia de las formas de crecimiento algales en la tipificación de un ambiente, Caracterización ficoflorística de los paredones de la Sierra de Juárez, 50: 121-133

Oaxaca, Ormosia (Leguminosae) in Mexico, including a new species from, 41:153-159

Oaxaqueño-poblana, Nueva especie de Casimiroa (Rutaceae) de la zona árida, 41:23-26

OBIETA, MA. CRISTINA y JOSE SARUKHAN. Estructura y composición la vegetación herbácea de un bosque uniespecífico de Pinus hartwegii I. Estructura y composición florística, 41:75-125

Observaciones sobre algunas especies mexicanas de Gnaphalium (Compositae), Notas taxonómicas y, 45:1521

OJEDA, LINA y BEATRIZ LUDLOW-WIECHERS. El polen del género Agave para la Península de Yucatán, 44:29-42
OJEDA, LINA, RODOLFO PALACIOS- CHAVEZ y BEATRIZ LUDLOW-WIECHERS. Consideraciones sobre la exina en Yucca, observada al microscopio de luz y microscopio de barrido, 48:153-154

Ormosia (Leguminosae) en México: 0 . panamensis, Una adición al género, 48:155-158

Ormosia (Leguminosae) in Mexico, including a new species from Oaxaca, 41:153-159

OROZCO-SEGOVIA, ALMA. Fisiología y ecología del fitocromo, su función en las semillas, 49:71-84

OROZCO-SEGOVIA, ALMA. Reseña de nuevos libros, 43:94-96

OROZCO-SEGOVIA, ALMA, BLANCA PEREZ-GARCIA y RAMON RIBA. El banco de esporas de helechos en el suelo de los Tuxtlas, Ver., 43:89-92

ORTIZ, EVANGELINA y JERZY RZEDOWSKI, Estudios quimiotaxonómicos de Bursera (Burseraceae). I. Bursera chemapodicta sp. n., 43:73-80

Ortografía correcta de Casimiroa calderonii Chiang et Medrano, Nota sobre la, 46:89

PALACIOS-CHAVEZ, RODOLFO, BEATRIZ LUDLOW-WIECHERS y LINA OJEDA. Consideraciones sobre la exina en Yucca, observada al microscopio de luz y microscopio de barrido, 48:153-154

PALACIOS-RIOS, MONICA y RAMON RIBA. Helechos de Veracruz: Adiantum (Pteridaceae), 44:43-62

Palinología de araliáceas, 45:117-132 
PALOMINO HASBACH, GUADALUPE. Análisis de la interacción entre radiaciones gamma y mms sobre la plántula de semillas de Triticale pretratadas con ácido ascórbico, 44:63-71

PALOMINO HASBACH, GUADALUPE, BALBINA VAZQUEZ BENITEZ, PATRICIA MARTINEZ AMERAYA Y PEDRO MERCADO RUARO. Estudios cromosómicos en seis especies mexicanas de comelináceas, 50:77-87

PALOMINO HASBACH, GUADALUPE, SOCORRO ZULETA LECHUGA y LEIA SCHEINVAR. Estudios citogenticos de dos especies y una variedad del género Nyctocereus (Cactaceae), 48:75-80

Pangola (Digitaria decumbens), Productividad primaria de tres praderas de especies tropicales: pará (Brachiaria mutica), grama amarga (Paspalum conjugatum) y, 41:-3-18

Papel de los aleloquímicos en el manejo de los recursos naturales, 49:85-98 Papel del microclima en la fisiología ecológica vegetal, El, 49:31-39

Pará (Brachiaria mutica), grama amarga (Paspalum conjugatum), Productividad primaria de tres praderas de especies tropicales:, 41:3-18

Parasitismo floral por abejas sociales (Meliponinae; Apidae) en el árbol quiropterófilo Crescentia alata (Bignoniaceae), 50:00-00.

Paspalum conjugatum y pangola (Digitaria decumbens), Productividad primaria de tres praderas de especies tropicales: pará (Brachiaria mutica), grama amarga, 41:3-18
PEDRAZA, R.A. y D.F. AUSTIN. Los géneros de Convolvulaceae en México, 44:3-16

PEDROCHE, FRANCISCO F., ABEL SENTIES G. y KURT M. DRECKMANN. Lista florística de las algas marinas bentónicas de la costa norte de Michoacán, México, 50:00-00 PEDROCHE, FRANCISCO F., KURT M. DRECKMANN y ABEL SENTIES G. La familia Rhodomelaceae (Ceramiales, Rhodophyta) en la costa del estado de Michoacán, México, 50:89-120

Península de Yucatán, El polen del género Agave para la, 44:29-42

Península de Yucatán, México. II., Musgos de la, 43:35-37

Península de Yucatán, Vegetación insular de la, 45:23-37

PERALES RIVERA, HUGO R. y JOSE GUADALUPE GARCIA FRANCO. Nota sobre la propagación y pérdida de viabilidad de las semillas de Chiranthodendron pectadactiylon Larr. (Sterculiaceae), 50:157-159

PERALES RIVERA, HUGO R. y JUAN ISMAEL CALZADA. La preservación con alcohol de ejemplares colectados para herbario, 50 151-155

PEREZ ESCANDON, BLANCA E. y MIGUEL ANGEL VILLAVICENCIO. Spigelia longiflora (Mart. et. Gal.): actividad antialimentaria en tres especies de insectos fitófagos, 48:135-138

PEREZ GARCIA, BLANCA, ALMA OROZCO-SEGOVIA y RAMON RIBA. El banco de esporas de 
helechos en el suelo de los Tuxtlas, Ver., 43:89-92

PEREZ GARCIA, MARTHA. Reseña de nuevos libros, 43:94

PEREZ JIMENEZ, L. ALFREDO. Jatropha chamelensis (Euphorbiaceae), nueva especie de la costa de Jalisco, México, 42:35-39

PEREZ NASSER, NIDIA. Reseña de nuevos libros, 44:105-106

Perturbaciones naturales en un bosque mesofilo de montaña en Tamaulipas, Notas sobre las, 48:139-142

Phaseolus chiapasanus Piper (Leguminosae: Phaseolinae), Estudio del polen de, 43:25-34

Phaseolus coccineus L. II. Sistemas reproductivos, Biología floral de poblaciones silvestres y cultivadas de, 46:3-12

Photosynthetic characteristics of coastal and desert plants of California, Comparative, 42:19-33

Phytolaccaceae del suroeste de Puebla, México, Una especie nueva de Stegnosperma , 46:37-41

PINKAVA, DONALD J. Vegetation and flora of the Bolson of Cuatro Ciénegas region, Coahuila, Mexico: III Cactaceae to Compositae, 41:127151

Pinus in the Western United States and Mexico, The altitudinal distribution of the genus, 42:55-71

Pinus hartwegii I. Estructura y composición florística, Estructura y composición la vegetación herbácea de un bosque uniespecífico de, 41:75125

Plantae uxpanapae I. Colubrina johnstonii sp. nov. (Rhamnaceae),
44:81-90; II. Novedades en Violaceae y Scrophulariaceae, 45:133-140

Plantas acuáticas mexicanas I: Myriophyllum quitense H.B.K. (Haloragaceae), Registros nuevos de, 45:147-149; II Luziola subintegra Swallen y L. spruceana Benth. ex Doell. (Gramineae), 46:90-91

Plantas superiores, Fotosíntesis y economía del carbono en, 49:41-60

Plants of California, Comparative photosynthetic characteristics of coastal and desert plants of, 42:19-33

Plántula de jemillas de Triticale pretratadas con ácido ascórbico, Análisis de la interacción entre radiaciones gamma y mms la, 44:63-71 Poblaciones silvestres y cultivadas de Phaseolus coccineus L. II. Sistemas reproductivos, 46:3-12

Polen de Phaseolus chiapasanus Piper (Leguminosae: Phaseolinae), Estudio del, 43:25-34

Polen del género Agave para la Península de Yucatán, El, 44:29-42

Polen en la región de San Luis Potosí, México, Interpretaciones sobre la lluvia de, 46:53-74

Polinización, Reducción en la fecundidad en Manfreda brachystachya (Cav.) Rose, una agavácea polinizada por murciélagos: los riesgos de la especialización en la, 48:147-149

Pontederia rotundifolia, Nuevo registro para México de, 41:161

Praderas de especies tropicales: pará (Brachiaria mutua), grama amarga (Paspalum conjugatum) y pangola (Digitaria decumbens), Productividad primaria de tres, 41:3-18 
Presevación con alcohol de ejemplares colectados para herbario, La, 50:151

Productividad primaria de tres praderas de especies tropicales: pará (Brachiaria mutica), grama amarga (Paspalum conjugatum) y pangola (Digitaria decumbens), 41:3-18

Propagación y pérdida de viabilidad de las semillas de Chiranthodendron pectadactylon Larr. (Sterculiaceae), Nota sobre la, 50:157-159

Psychotria (Rubiaceae, Psychotrieae), New taxa in Mexican, 47:49-64

Psychotrieae, New taxa in Mexican Psychotria Rubiaceae, 47:49-64

Pteridaceae, Helechos de Veracruz: Adiantum, 44:43-62

PUCHET, C., M. RAMIREZ, R. VILLALON, E. ZAPATA, LILA LORENZO, A. RAMIREZ ROA, M.A. SOTO ARENAS, A. BRECEDA, M. DEL C. CALDERONy C. CORTEZ. Notas sobre la fitogeografía del bosque mesófilo de montaña en la Sierra Madre del Sur, México, 44:97-102

Puebla, México, La vegetación acuática vascular de seis lagos-cráter del estado de, 46:75-88

Puebla, México, Una especie nueva de Stegnosperma (Phytolaccaceae) del suroeste de, 46:37-41

PULIDO, MA. TERESA P. y STEPHEN D. Koch. Inventario florístico en el cerro Tetzcotzinco, Texcoco, Estado de México, 48:81-94 Quimiotaxonomía de Bursera, 43:73-80 Radiaciones gamma y mms sobre la plántula de semillas de Triticale pretratadas con ácido ascórbico,
Análisis de la interacción entre, 44:6371

RAMAMOORTHY, T.P., P. HIRIART VALENCIA y FRANCISCO GONZALEZ-MEDRANO. Neoeplingia Ramamoorthy, Hiriarat Medrano (Labiatae) un nuevo género de Hidalgo, México, 43:61-65

RAMIREZ, M., R. VILLALON, E. ZAPATA, LILA LORENZO, A. RAMIREZ ROA, M.A. SOTO ARENAS, A. BRECEDA, M. DEL C. CALDERON, H. CORTEZ y C. PUCHET. Notas sobre la fitogeografía del bosque mesófilo de montaña en la Sierra Madre del Sur, México, 44:97-102

RAMIREZ GARCIA, PEDRO y ALEJANDRO NOVELO RETANA. La vegetación acuática vascular de seis lagos-cráter del estado de Puebla, México, 46:75-88

RAMIREZ ROA, A., M.A. SOTO ARENAS, A. BRECEDA, M. DEL C. CALDERON, H. CORTEZ, C. PUCHET, M. RAMIREZ, R. VILLALON, E. ZAPATA y LILA LORENZO. Notas sobre la fitogeografía del bosque mesófilo de montaña en la Sierra Madre del Sur, México, 44:97-102

Randia (Rubiaceae, Gardenieae), New taxa and new name in Mexican and Central American, 48:37-48

Rango de hospedantes de Rhynchosporium secalis, 46:19-24

Reconocimiento florístico y consideraciones fitogeográficas del bosque mesófilo de montaña de Teocelo, Veracruz, 48:35-63 
Recursos naturales, Papel de los aleloquímicos en el manejo de los, 49:85-98

Reducción en la fecundidad en Manfreda brachystachya (Cav.) Rose, una agavácea polinizada por murciélagos: los riesgos de la especialización en la polinización, 48:147-149

Registro para México de Gleditsia (Leguminosae), Nuevo, 48:143-144

Registros de Juncaceae y Liliaceae en el Valle de México, Nuevos, 46:25-27

Registros nuevos de plantas acuáticas mexicanas I: Myriophyllum quitense H.B.K. (Haloragaceae), 45:147-149; II Luziola subintegra Swallen y $L$. spruceana Benth. ex Doell. (Gramineae), 46:90-91

Registros y nuevas combinaciones de gramíneas del Valle de México, Nuevos, 48:19-22

República Mexicana, Nuevos registros de ciperáceas para la flora del Valle de México y de la, 44:17-21

Rhamnaceae Plantae uxpanapae I. Colubrina johnstonii sp. nov. 44:81-90 Rhamnaceae una planta muy poco colectada, Condalia velutina, 45:143-146 Rhizophora harrisonii (Rhizophoraceae), un nuevo registro para las costas de México, 41:163-165

Rhizophoraceae, un nuevo registro para las costas de México, Rhizophora harrisonii, 41:163-165.

Rhodomelaceae (Ceramiales, Rhodophyta) en la costa del estado de Michoacán, México, La familia, 50:89 $-120$

Rhynchosporium secalis en el mumủo y en México, Distribución de, 48:3-11
Rhynchosporium secalis, Rango de hospedantes de, 46:19-24

RIBA RAMON. Reseña de nuevos libros, 43:93; 44:103-104; 50:00-00.

RIBA, RAMON y MA. EUGENIA FRAILE. Distribución esporangial en estróbilos de especies de Selaginella, 41:33-40

RIBA, RAMON y MONICA PALACIOS- RIOS. Helechos de Veracruz: Adiantum (Pteridaceae), 44:43-62

RIBA, RAMON, BLANCA PEREZGARCIA y ALMA OROZCOSEGOVIA. El banco de esporas de helechos en el suelo de los Tuxtlas, Ver., 43:89-92

RICO ARCE, MA. DE LOURDES, Acacia velvae y $A$. Mirandae (Leguminosae), dos nuevas especies para México, 43:67-71

RICO GRAY, VICTOR. Rhizophora harrisonii (Rhizophoraceae), un nuevo registro para las costas de México, 41:163-165

RINCON, EMANUEL y PILAR HUANTE. Nutrición mineral, 49:717

Rubiaceae, Dos nuevas especies mexicanas de la familia, 44:73-80

Rubiaceae, en la región de Los Tuxtlas, Veracruz, México, La familia, 50:0000.

Rubiaceae, Gardenieae, New taxa and new name in Mexican and Central American Randia 47:37-48

Rubiaceae, In Mexico, with description of a new species, E. uxpanapensis, First record of Elaeagia, 45:65-69

Rubiaceae, Psychotrieae, New Taxa in Mexican Psychotria, 47:49-64 
RUDD, VELVA E. Ormosia (Legumiosae) in Mexico, including a new species from Oaxaca, 41:153-159 RUDD, VELVA E. Una adición al género Ormosia (Leguminosae) en México: O. panamensis, 48:155-158

Rutaceae de Tabasco y Veracruz, Una nueva especie de Decazyx, 43:1-3

Rutaceae de la zona árida oaxaqueñopoblana, Nueva especie de Casimiroa, 41:23-26

RZEDOWSKI, JERZY. Algunas adiciones al género Acourtia (Compositae-Mutisieae), 45:97-109

RZEDOWSKI, JERZY. Dos especies nuevas de Compositae-Astereae del Valle de México, 45:111-116

RZEDOWSKI, JERZY. Dos nuevas especies mexicanas de la familia Rubiaceae, 44:73-80

RZEDOWDKI, JERZY y EVANGELINA ORTIZ, Estudios quimiotaxonómicos de Bursera (Burseraceae). I. Bursera chemapodicta sp. n., 43:73-80

RZEDOWSKI, JERZY y ROSA MA.

VEGA. Nota aclaratoria sobre las hojas de Valeriana vaginata (Valerianaceae), 41:167-170

RZEDOWSKI, JERZY y SOCORRO GONZALEZ E. Algunos aspectos ecológicos y fitogeográficos de las especies de Cyperaceae en el Valle de México. I. Afinidades ecológicas, 45:39-47; II. Análisis fitogeográfico, 46:29-35

RZEDOWSKI, JERZY, EMILY J. LOTT y VICTOR JARAMILLO L. Un género nuevo de la parte meridional de México: Gypsacanthus
(Acanthaceae, Justicieae, Odontoneminae), 46:47-51

Rzedowskia, un nuevo género de Celastraceae de México, 41:41-46

San Luis Potosí, México, Interpretaciones sobre la lluvia de polen en la región de, 46:53-74

SANCHEZ VEGA, ISIDORO y STEPHEN D. KOCH. Estudio biosistemático de Eragrostis mexicana, $E$. neomexicana, $E$. ocurttiana y E. virescens (Gramineae: Chloridoideae), 48:95-112

SARUKHAN, JOSE y ALBERTO BURQUEZ. Biología floral de poblaciones silvestres y cultivadas de Phaseolus coccineus L. II. Sistemas reproductivos, 46:3-12

SARUKHAN, JOSE y CRISTINA OBIETA. Estructura y composición la vegetación herbácea de un bosque uniespecífico de Pinus hartwegii I. Estructura y, 41:75-125

Savia sessiliflora (Euphorbiaceae) en México, Nota sobre la distribución de, 47:90-91

SCHEINVAR, LEIA, GUADALUPE PALOMINO HASBACH Y SOCORRO ZULETA. Estudios citogenéticos de dos especies y una variedad del génern Nyctocereus (Cactaceae), 48:75

SCHULZE, ERNST-DETLEF, HAROLD A. MOONEY, STEPHEN H. BULLOCK, y ANA MENDOZA. Water contents of wood of tropical deciduous forest species during the dry season, 48:113-118

Scrophulariaceae, Plantae uxpanapae II. novedades en Violaceae y, 45:133-140 
Scutellaria (Labiatae) de Hidalgo, México, Una nueva especie de, 46:4346

Selaginela heterófila del estado de Guerrero, México. Selaginella rzedowskii, una nueva especie de, 44:23-27

Selaginella Distribución esporangial en estróbilos de especies de, 41:33-40

Selaginella rzedowskii, una nueva especie de selaginela heterófila del estado de Guerrero, México, 44:23-27

Selva húmeda tropical de los Tuxtlas Veracruz, Especies CAM en la, 45:150-153

Semillas de Chiranthodendron pectadactylon Larr. (Sterculiaceae), Nota sobre la propagación y pérdida de viabilidad de las, 50:157-159

Semillas en la conservación de especies vegetales, El almacenamiento de, 49:61-69

Semillas, Fisiología y ecología del fitocromo, su función en las, 49:71-84 SENTIES G., ABEL, FRANCISCO F. PEDROCHE y KURT M. DRECKMANN. La familia Rhodomelaceae (Ceramiales, Rhodophyta) en la costa del estado de Michoacán, México, 50:89-120

SENTIES G., ABEL, KURT M. DRECKMANN y FRANCISCO F. PEDROCHE. Lista florística de las algas marinas bentónicas de la costa norte de Michoacán, México, 50: 19 $1-42$

Sierra de Juárez, Oaxaca, Importancia de las formas de crecimiento algales en la tipificación de un ambiente, Caracterizacíon ficoflorística de los paredones de la, 50: 121-133
Sierra Madre del Sur, México, Notas sobre la fitogeografía del bosque de montaña en la, 44:97-102

Sistemas reproductivos, Biología floral de poblaciones silvestres y cultivadas de Phaseolus coccineus L. II, 46:3-12

Sobre el significado del "co" en coevolución, 44:91-94

Sobre la ecología reproductiva de Nicotiana glauca Grah: una maleza de distribución cosmopolita, 41:47-73

Solanaceae de Norteamérica, Fstudios cromosómicos en Lycium, 43:9-23

Solanaceae de Norteamérica, Nuevas combinaciones y una nueva variedad de Lycium, 43:5-8

Solanaceae, El número cromosómico de Grabowskia geniculata, 45:141-142

Solanaceae, El número cromosómico de Lycium leiospermum I.M. Johnston, 48:145-146

SOSA, VICTORIA. Características palinológicas de las araliáceas de México. 45:117-132

SOTO ARENAS, M.A., A. BRECEDA, M. DEL C. CALDERON, H. CORTEZ, C. PUCHET, M. RAMIREZ, R. VILLALON, E. ZAPATA y LILA LORENZO. Notas sobre la fitogeografía del bosque mesófilo de montaña en la Sierra Madre del Sur, México, 44:97-102

Spigelia longiflora (Mart. et. Gal.): actividad antialimentaria en tres especies de insectos fitófagos, 48:135138

Stegnosperma (Phytolaccaceae) del suroeste de Puebla, México, Una especie nueva de, 46:37-41

Sterculiaceae, Nota sobre la propagación y pérdida de viabilidad de las semillas 
de Chiranthodendron pectadactylon Larr., 50: 157-159

SUAREZ RAMOS, GUADALUPE y E. MARK ENGLEMAN. Estudio de los canales resiníferos de la corteza de Bursera copallifera y Bursera grandifolia, 42:41-54

Tabasco, México, Algunos musgos de, 48:13-18

Tabasco y Veracruz, Una nueva especie de Decazyx (Rutaceae) de, 43:1-3

Tamaulipas, México, Mimosa martindelcampoi Medrano (Leguminosae), una especie nueva del sudoeste de, 43:3942

Tamaulipas, Notas sobre las perturbaciones naturales en un bosque mesófilo de montaña en, 48:139-142

Tauschia (Umbelliferae) del Estado de México, Una nueva especie de, 41:1921

TAVERA SIERRA, ROSA LUZ y JORGE GONZALEZ GONZALEZ. Caracterización ficoflorística de los paredones de la Sierra de Juárez, Oaxaca. Importancia de las formas de crecimiento algales en la tipificación de un ambiente, 50:121

Tehuacán-Cuicatlán, Análisis de la vegetación arbórea en la provincia floristíca de, 45:49-64

Tehuacán-Cuicatlán, Fitogeografía del Valle de, 50:135-149

TELLEZ VALDEZ, OSWALDO. Nota sobre la distribución de Carpodiptera ameliae (Tiliaceae), en México, 41:166

TELLO BALDERAS, J. JESUS y EDMUNDO GARCIA MOYA. El maguey (Agave, subgénero Agave) en el altiplano potosino-zacatecano, 48:119-134

Teocelo, Veracruz, Reconocimiento florístico y consideraciones fitogeográficas del bosque mesófilo de montaña de Teocelo, Veracruz, 48:35-63

Tepescohuite en México, Nota sobre la identidad del, 48:151-152

Tetzcotzinco, Texcoco, Estado de México, Inventario florístico en el cerro, 48:81-94

Texcoco, Estado de México, Inventario florístico en el cerro Tetzcotzinco, 48:81-94

Tigridia martinezii, una especie nueva de iridáceas del estado de Hidalgo (México), 47:3-6

Tiliaceae, en México, Nota sobre la distribución de Carpodiptera ameliae, 41:166

TINOCO OJANGUREN, CLARA y CARLOS VAZQUEZ-YANES. Especies CAM en la selva húmeda tropical de los Tuxtlas, Veracruz, 45:150-153

TOLEDO, JORGE R. y CARLOS VAZQUEZ YANES. El almacenamiento de semillas en la conservación de especies vegetales, 49:61-69

Triticale pretratadas con ácido ascórbico, Análisis de la interacción entre radiaciones gamma y mms sobre la plántula de semillas de, 44:63-71

Tuxtlas, Ver. El banco de esporas de helechos en el suelo de Los, 43:89-92

Tuxtlas, Veracruz. Especies CAM en la selva húmeda tropical de, Los, 45:150153 
Tzonpotónic mencionado por Francisco Hernández en su Historia Natural de la Nueva España, Nota sobre la identidad del segundo, 47:87-89

Umbelliferae del Estado de México, Una nueva especie de Tauschia, 41:19-21

United States and Mexico, The altitudinal distribution of the genus Pinus in the, 42:55-71

VALDES, MARIA. Aspectos ecofisiológicos de las micorrizas, 49:19-30

Valeriana vaginata (Valerianaceae), Nota aclaratoria sobre las hojas de, 41:167-170

Valerianaceae, Nota aclaratoria sobre las hojas de Valeriana vaginata, 41:167-170

Valle de México, Adiciones a la flora arvense del, 41:27-32

Valle de México, Dos especies nuevas de Compositae-Astereae del, 45:111-116 Valle de México, I. Afinidades ecológicas, Algunos aspectos ecológicos y fitogeográficos de las especies de Cyperaceae en el, 45:3947; II. Análisis fitogeográfico, 46:2935

Valle de México, Nota sobre tres especies de ciperáceas del, 44:95-96

Valle de México, Nuevos registros de Juncaceae y Liliaceae en el, 46:25-27

Valle de México, Nuevos registros y nuevas combinaciones de gramíneas del, 48:19-22

Valle de México, y de la República Mexicana, Nuevos registros de ciperáceas para la flora del, 44:17-21

Valle de Tehuacán-Cuicatlán, Fitogeografía del, 50: 135-149
VAZQUEZ BENITEZ, BALBINA, PATRICIA MARTINEZ ALMERAYA, PEDRO MERCADO RUARO y GUADALUPE PALOMINO HASBACH. Estudios cromosómicos en seis especies mexicanas de comelináceas, 50:77-87

VAZQUEZ YANES, CARLOS. Fisiología ecológica de plantas, La, 49:3-5

VAZQUEZ YANES, CARLOS y CLARA TINOCO OJANGUREN. Especies CAM en la selva húmeda tropical de los Tuxtlas, Veracruz, 45:150-153

VAZQUEZ YANES, CARLOS y JORGE R. TOLEDO. El almacenamiento de semillas en la conservación de especies vegetales, 49:61-69

VEGA, ROSA MA. y JERZY RZEDOWSKI. Nota aclaratoria sobre las hojas de Valeriana vaginata (Valerianaceae), 41:167-170

Vegetación acuática vascular de seis lagos-cráter del estado de Puebla, México, La, 46:75-88

Vegetación arbórea en la provincia florística de Tehuacán-Cuicatlán, Análisis de la, 45:49-64

Vegetación herbácea de un bosque uniespecífico de Pinus hartwegii I.

Estructura y composición florística, Estructura y composición la, 41:75125

Vegetación insular de la Península de Yucatán, 45:23-37

Vegetation and flora of the Bolson of Cuatro Ciénegas region, Coahuila, Mexico: III Cactaceae to Compositae, 41:127-151 
Veracruz, Adiantum (Pteridaceae), Helechos de, 44:43-62

Veracruz, México, La familia Rubiaceae en la región de Los Tuxtlas, 50:43-68 Veracruz, Una nueva especie de Decazyx (Rutaceae) de Tabasco y, 43:1-3

VILLALON, R., E. ZAPATA, LILA LORENZO, A. RAMIREZ ROA, M.A. SOTO ARENAS, A. BRECEDA, M. DEL C. CALDERON, H. CORTEZ, C. PUCHET y M. RAMIREZ. Notas sobre la fitogeografía del bosque mesófilo de montaña en la Sierra Madre del Sur, México, 44:97-102

VILLASEÑOR, JOSE LUIS. Clave genérica para las compuestas de la cuenca del río Balsas, 47:65-86

VILLASEÑOR JOSE LUIS. Reseña de libros, 47:95-96

VILLASEÑOR, JOSE LUIS, PATRICIA DAVILA y FERNANDO CHIANG. Fitogeografía del Valle de Tehuacán-Cuicatlán, 50:135-149

VILLAVICENCIO, MIGUEL ANGEL y BLANCA E. PEREZ ESCANDON. Spigelia longiflora (Mart. et. Gal.): actividad antialimentaria en tres especies de insectos fitófagos, 48:135-138

VILLERS, LOURDES, ISOLDA LUNA, LUCIA ALMEIDA y LILA LORENZO. Reconocimiento florístico y consideraciones fitogeográficas del bosque mesófilo de montaña de Teocelo, Veracruz, 48:35-63

Violaceae y Scrophulariaceae, Plantae uxpanapae II. Novedades en, 45:133140
Water contents of wood of tropical deciduous forest species during the dry season, 48:113-118

WENDT, TOM. Plantae uxpanapae I. Colubrina johnstonii sp. nov. (Rhamnaceae), 44:81-90; II. Novedades en Violaceae y Scrophulariaceae, 45:133140

Wood of tropical deciduous forest species during the dry season, Water contents of, 48:113-118

YEATON, RICHARDI. The altitudinal distribution of the genus Pinus in the Western United States and Mexico, 42:55-71

Yucatán, El pólen del género Agave para la Península de, 44:29-42

Yucatán, México. III., Musgos de la Península de, 43:35-37 Yucatán, Vegetación insular de la Península de, 45:23-37

Yucca, observada al microscopio de luz y microscopio de barrido, Consideraciones sobre la exina en, 48:153154

Zacatecas, México. II. Musgos de, 46:1318; III. Síntesis y fitogeografía, 47:1324

ZAMUDIO, SERGIO y CLAUDIO DELGADILLO M. Algunos musgos de Tabasco, México, 48:13-18

ZAPATA, E., LILA LORENZO, A. RAMIREZ ROA, M.A. SOTO ARENAS, A. BRECEDA, M. DEL C. CALDERON, H. CORTEZ, C. PUCHET, M. RAMIREZ y R. VILLALON. Notas sobre la fitogeografía del bosque de montaña en la Sierra Madre del Sur, México, 44:97-102 
Zona árida oaxaqueño-poblana, Nueva especie de Casimiroa (Rutaeae) de la, 41: $23-26$

ZUCHOWSKI, WILLOW, LINCOL P. BROWER y WILLIAM $\mathrm{H}$. CALVERT. The impact of forest thinning on microclimate in monarch butterfly (Danaus plexippus L.) overwintering areas of Mexico, 42:11-18
ZULETA LECHUGA, SOCORRO, LEIA SCHEINVAR y GUADALUPE PALOMINO HASBACH. Estudios citogenéticos de dos especies y una variedad del género Nyctocereus (Cactaceae), 48:75-80 


\section{INDICE DE TAXA Y TEMAS}

abejas sociales, robo de polen por véase Crescentia alata

Acacia véase Leguminosae

Acanthaceae

Gypsacanthus gen. nov. Puebla, 46:47-51.

nelsonii sp. nov. Puebla, 46:47-51.

Acourtia véase Compositae

Adiantum véase Pteridaceae

Agavaceae

Agave, Yucatán polen del género

para, 44:29-42.

subg. Agave, en el altiplano Potosino- Zacatecano, 48:119-134.

Manfreda

brachystachya, polinizada por murciélagos, 48:147- 149.

\section{Yucca}

elephantipes exina, 48:153-154.

filifera, exina, 48:153-154.

Agave véase Agavaceae

Algae

Michoacán, lista florística, 50:19-42

Rhodomelaceae, en, 50:89-120

Oaxaca,

Sierra de Juárez, paredones de la, ficoflorística, 50: 121-133

Aleloquímica véase Ecofisiología

Allosidastrum véase Malvaceae

Andropogon véase Gramineae

Aralia véase Araliaceae

Araliaceae, México, palinología, 45:117

132.

Aralia, palinología, 45:117- 132.

Dendropanax, palinología, 45:117-132.

Didymopanax, palinología, 45:117-132.

Hedera, palinología, 45:117-132.

Oreopanax, palinología, 45:117-132.

Sciadodendron, palinología, 45:117-132 árbol de piel véase Mimosa tenuiflora Asphodelus véase Liliaceae

Baccharis véase Compositae

Bibliografia

reseña de libros, 43:93-96;44:103; 106;46:93- 94;47:95-96;48:159;

50: $161-165$

Bignoniaceae

Crescentia alata, robo de polen por, abejas sociales, 50:69-76

Biología floral véase Phaseolus coccineus

Bolsón de Cuatro Ciénegas véase Cuatro Ciénegas

Boraginaceae

Bourreria, madera, ultraestructura, 45:3-14.

Cordia, madera, ultraestructura, 45:3-14.

Bosque

mesófilo de montaña véase también (Michoacán; Sierra Madre del Sur; Tamaulipas; Teocelo)

tropical caducifolio especies leñosas, contenido de agua, 48:113-118.

Bourreria véase Boraginaceae

Brachiaria véase Gramineae

Bryophyta

musgos

alpinos del Nevado de Colima, 47:7-11

Tabasco, 48:13-18

Yucatán, 43:35-37

Zacatecas, 46:13 18; 47: 13- 24.

Bursera véase Burseraceae

Burseraceae

Bursera quimiotaxonomía, 43:73-80. chanapodicta sp. nov., Guerrero, 43:73-80. 
copallifera, anatomía de los canales resiníferos, 42:41-54.

\section{Cactaceae}

Nyctocereus, citogenética, 48:75-80.

California véase Estados Unidos de

América

Carpodiptera véase Tiliaceae

Casasia véase Rubiaceae

Casimiroa véase Rutaceae

Celastraceae

Rzedowskia gen. nov., 41:41-46.

tolantonguensis sp. nov., Hidalgo, 41: 41-46.

Cerro de Tetzcotzinco véase Tetzcotzinco

Chiapas véase Randia matudae nom. nov.

Chiranthodendron véase Sterculiaceae

Cnidoscolus véase Euphorbiaceae

Coahuila

Cuatro Ciénegas, Bolsón de, vegetación y flora, 41:127-151.

Co-evolución, significado del "co" en, 44:91-94.

Coleoptera

Epilachna

vevivestis véase Spigelia longiflora,

e insectos fitófagos

Colubrina véase Rhamnaceae

Comelinaceae

estudios cromosómicos en especies de, 50:77-87

Compositae

Cuenca del Río Balsas, clave genérica, 47:65-86.

Acourtia

bravohollisiana sp. nov., Guerrero, 45:97-109.

discolor sp. nov., Oaxaca, 45:97-109.

fragans sp. nov., Puebla, 45:97-109.

huajuapana var. actinomorpha var.

$$
\text { nov., 45:97-109. }
$$

matudae sp. nov., Estado de México, 45:97-109.

Astranthium

reichei $\mathrm{sp}$. nov., Estado de México, 45:111-116.

Baccharis

erosoricola sp. nov., Estado de México, 45:111-116.

Gnaphalium, notas taxonómicas, 45:

15-21.

oxyphyllum var. nataliae var. nov., 45: 15-21.

Computadoras, aplicación de, véase

México, flora

Condalia véase $\mathrm{Rhamnaceae}$

Convolvulaceae

México, clave para géneros, 44:3-16.

Cordia véase Boraginaceae

Crescentia véase Bignoniaceae

Cuatro Ciénegas véase Coahuila

Cuenca del Río Balsas véase

Compositae

Cyperaceae

Valle de México

ecología y fitogeografía, 45:39-47; 46:29-35.

nuevos registros, 44:17-21.

Eleocharis, especies en el Valle de

México, 44:95-96.

Danaidae

Danaus

plexippus, impacto de la deforestación, área de invernación, 42:11-18

Danaus véase Danaidae

Decazyx véase Rutaceae

Dendropanax véase

Araliaceae

Didymopanax véase Araliaceae

Didymae véase Rubiaceae

Digitaria véase Gramineae

Echeandia véase Liliaceae

Ecofisiología

Aleloquímica, 49:85-98. 
fitocromo, 49:71-84.

fotosíntesis, 49:41-60.

micorrizas, 49:19-30.

microclima, 49:31-39.

nutrición mineral, 49:7-17.

plantas, 49:3-5.

semillas, 49:61-69.

Eichhornia véase Pontederiaceae

Elaeagia véase Rubiaceae

Eleocharis véase Cyperaceae

Epilachna véase Coleoptera

Eragrostis véase Gramineae

Estado de México véase México, Estado de,

Estados Unidos de América véase también (Pinus, distribución altitudinal)

California

Valle de la muerte, características

fotosintéticas de plantas nativas,

42:19-33.

Euphorbiaceae

\section{Cnidoscolus}

spinosus, en Jalisco, componentes

fenológicos, 42:1-9.

\section{Jatropha}

chamelensis sp. nov., Jalisco, 42:35-39

Savia

sessiliflora, México, distribución, 47:90 91.

Flora

México, aplicación de computadoras, 48:65-73.

Valle de, arvense, adiciones, 41: 27-32.

Fitocromo véase Ecofisiología Fotosíntesis véase Ecofisiología

Fungi

Rynchosporium

secalis distribución, 48:3-11; hospedantes, 46:19-24.
Gleditsia véase Leguminosae Gnaphalium véase Compositae Grabowskia véase Solanaceae grama amarga véase Paspalum conjugatum

Gramineae

Valle de México, nuevos registros, 48:19:22.

Andropogon var. brevipedicellatus comb. nov., 48: 19-22. var. sanguineus comb. nov., 48:19-22. Brachiaria mutica, productividad primaria, 41:3-18.

\section{Digitaria} decumbens, productividad primaria, 41:3-18.

Eragrostis mexicana, variación morfológica, 48:95-112.

subesp. virescens, número cromosómico, 48:95-112.

neomexicana, variación morfológica, 48:95-112.

orcutiana, variación morfológica, 48: 95-112.

Luziola

spruceana, Tabasco, nuevo registro, 46:90-91.

subintegra, Tabasco, nuevo registro, 46:90-91.

Muhlenbergia

orophila, Valle de México, nuevo registro, 48:19-22.

Panicum

miliaceum, Valle de México, nuevo registro, 48:19-22.

Paspalum

conjugatum, productividad primaria, 41:3-18.

Setaria 
faberi, Valle de México, nuevo registro, 48:19-22.

Triticale, semillas, plántulas, radiaciones gama y ácido ascórbico sobre, 44:63-71.

granjeno véase Condalia velutina granjeno rojo véase Condalia velutina Guanajuato véase Mimosa biuncifera; Mimosa monancistra.

Guerrero véase Acacia velvae sp. nov.; Acourtia bravohollisiana, sp. nov.; Bursera chemapodicta sp. nov.;

Selaginella rzedowski sp. nov.

Gypsacanthus véase Acanthaceae Haloragaceae

Myriophyllum quitense, Estado de México, nuevo registro, 45:147-149.

Hedera véase Arialiaceae

Helechos véase Pteridophyta

Herbario preservación de ejemplares, 50:151-155

Herbivoría

aparato para el estudio de, 43:81-88. Hernández, Francisco

segundo Tzonpotónic, mencionado en su Historia Natural, 47:87-89.

Hidalgo véase Neoeplingia leucophylloides sp. nov.; Rzedowskia tolantoguensis sp. nov.; Scutellaria molanguitensis sp. nov.; Tigridia martinezii sp. nov.

Iridaceae

Tigridia

martinezii, sp. nov., Hidalgo, 43:3-6. Jalisco véase también (Cnidoscolus spinosus Jatropha chamelensis sp. nov.) Nevado de Colima véase musgos Jatropha véase Euphorbiaceae Juncaceae

\section{Juncus}

arcticus var. andicola, Valle de México, nuevo registro, 46:25-28. bufonius, Valle de México, nuevo registro, 46:25-28.

Juncus véase Juncaceae

Kickxia véase Scrophulariaceae

Labiatae

\section{Lamium}

amplexicaule, en el Valle de México, 41:27-32

purpureum, en el Valle de México, 41:27-32.

Neoplingia gen. nov., 43:61-65.

leucophylloides sp. nov., Hidalgo 43: 61-65.

Scutellaria

molanguitensis sp. nov., Hidalgo, 46:43-46.

Lamium véase Labiatae

Leguminosae

Acacia

mirandae sp. nov., Oaxaca, 43:67-71.

velvae sp. nov., Guerrero, 43:67-71.

Gleditsia, México, nuevo registro, 48:143-144.

Mimosa, madera, anatomía, 50:3-17

biuncifera, Guanajuato, ecología 43:43-60.

martindelcampoi sp. nov., Tamaulipas, 43:39-42.

monancistra, Guanajuato, ecología, 43: 43-60.

tenuiflora, identidad taxonómica, $\quad 48$ : 151-152.

\section{Ornosia}

clave para las especies, 48:155-158. en México, 41:153-159.

oaxacana sp. nov., Oaxaca, 41: 153-159

panamensis, descripción y distribución, 48:155-158.

\section{Phaseolus}

chiapasanus, polen, 43:25-34. coccineus, biología floral, 46:3-12. Lepidoptera 


\section{Spodoptera}

frugipeda véase Spigelia longiflora, e insectos fitófagos.

\section{Pseudaletia}

unipunctatus véase Spigelia longiflora, e insectos fitófagos.

\section{Liliaceae}

\section{Asphodelus}

fistulosus, Valle de México, nuevo registro, 46:25-27.

\section{Echeandia}

durangensis, Valle de México, nuevo registro, 46:25-27.

Limnocharis véase Limnocharitaceae

Limnocharitaceae

Limnocharis laforestii lectotipificación, 47:92-93.

Lindernia véase Scrophulariaceae lirio acuático véase Eichhornia crassipes Longaniaceae

\section{Spigelia}

longiflora, e unsectos fitófagos, 48: 135-138

Los Tuxtlas véase Veracruz

Luziola véase Gramineae

Lycium véase Solanaceae

maguey véase Agave, subg. Agave

Malvaceae

Allosidastrum stat. nov., 48:23-34.

dolichophyllum sp. nov., Venezuela, 48:23-34.

hilarianum comb. nov., 48:23-34.

interruptum comb. nov., 48:23-34.

pyramidatum, comb. nov., 48:23-34.

Manfreda véase Agavaceae

mariposa monarca véase Danus

plexippus

membrillo cimarrón véase Condalia velutina

México véase Araliacea; Carpodiptera ameliae; Convolvulaceae; Flora, aplicación de computadoras; Gleditzia, nuevo registro; Lindernia antipoda, nuevo registro; Pontederia rotundifolia; Rhizophora harrison; Savia sessiliflora México, Estado de, véase también (Acourtia matudae sp. nov.; Astranthium reichei sp. nov.; Baccharis erosoricola sp. nov.; Myriophyllum quitense, nuevo registro Tauschia neglecta sp. nov.)

Texcoco

Tetzcotzinco (cerro de), inventario florístico, 48:81-94.

México, Valle de, véase también (Asphodelus fistulosus, nuevo registro Astranthium reichei sp. nov.; Baccharis erosoricola sp. nov.; Cyperaceae; Echeandia durangensis, nuevo registro Eleocharis ssp.; Gramineae, nuevos registros; Juncaceae; Juncus articus var. andicola, nuevo registro; Kickia elatine ssp. crinita; Lamium amplexicaule; Lamium purpureum; Liliaceae; Muhlenbergia orophila, nuevo registro; Panicum mileaceum, nuevo registro; Polygonum convolvulus; Setaria fabril, nuevo registro).

flora arvense, adiciones, 41:27-32.

Michoacán véase también (Algae; Rhodomelaceae)

bosque mesófilo de montaña, ecología reproductiva, 47:25-35.

Micorriza véase Ecofisiología

Mimosa véase Leguminosae

Musgos véase Bryophyta

Muhlenbergia véase Gramineae Myriophyllum véase Halorangaceae Neoepligia véase Labiatae Nevado de Colima véase Jalisco

Nicotiana véase Solanaceae

Nutrición mineral véase Ecofisiología

Nictocereus véase Cactaceae

Nymphaea véase Nymphaeaceae

Nymphaeaceae Nyphaea 
gracilis, esclarecimiento taxonómico, 45:85-95.

Oaxaca véase Acacia mirandae sp. nov.; Acourtia discolor sp. nov.: Casimiroa calderonii sp. nov.; Elaeagia Uxpanapensis sp. nov.; Ormosia oaxacana sp. nov.; Psychotria izabalensis subesp. oaxacana subesp. nov.; Psychotria megalatha sp. nov.

Sierra de Juárez véase Algae

Oreopanax véase Araliaceae Ormos:a véase leguminosae pangola véase Digitaria decumbens

Panicum véase Gramineae pará véase Brachiaria mutica Paspalum véase Gramineae Phaseolus véase Leguninosae Pytolaccaceae

\section{Stegnosperma}

sanchezii sp. nov., Puebla, 46:37-41 pico de pájaro véase Condalia velutina Pinaceae

Pinus, Estados Unidos de América, distribución altitudinal, 42:55-71. hartwegii, bosque, estructura y composición florística, 41: 75-125.

Pinus véase Pinaceae

Plantae Uxpanapae véase Colubrina johnstonii sp. nov.; Rinorea uxpanapa sp. nov.; Uroskinnera hirtiflora var. brevilopa var. nov.; Scrophulariaceae; Violaceae

Plantas (fisiología ecológica) véase Ecofisiología acuáticas véase Eichhomia crassipes, demografía y crecimiento; Limnocha ris laforestii, lectotipificación; Luziola spruceana, tabasco, nuevo registro; Luziona subintegra, Tabasco, nuevo registro; Myriophyllum quitense, Esta do de México, nuevo registro; Nymphaea gracilis, esclarecimiento taxonómico; Pontederia rotundifolia, México, nuevo registro.
Poloygonaceae

Polygonum

convulvulus, Valle de México, 41:27-

32.

Polygonum véase Polygonaceae

Pontederia véase Pontederiaceae

Pontederiaceae

Eichhornia

crassipes, demografía y crecimiento, 45:71-83.

Pontederia rotundifolia, México, nuevo registro 41:161.

Psychotria véase Rubiaceae

Pteridaceae véase Pteridophyta

Pteridophyta

helechos, los Tuxtlas, banco de esporas, 43:89-92.

Pteridaceae

Adiantum, de Veracruz, clave para las especies, 44:43-62.

Selaginella, esporangios en estróbilos, 41:33-40.

rzedowski sp. nov., Guerrero, 44:23-

27.

Puebla véase también (Acourtia fragans sp. nov.; Gypsacanthus nelsonii sp. nov.; Stegnosperma sanchezii, sp. nov.) vegetación acuática, de lagos cráter, 46:75-88.

Querétaro véase Randia hidalguensis sp. nov.

Randia véase Rubiaceae

Rhamnaceae

Colubrina

johnstonii sp. nov., Veracurz, 44:8190.

\section{Condalia}

velutina, nombres comunes, usos, distribución geográfica 45:143-146. Rhizophora véase Rhizophoraceae Rhizophoraceae

Rhizophora 
harrisonni, México, nuevo registro, 41:163-165.

Rhodomelaceae véase Algae Rhynchosporium véase Fungi

Richardia véase Rubiaceae

Rinorea véase Violaceae

Río Balsas, Cuenca de, véase Compositae

Rubiaceae, en los Tuxtlas, 50:43-68

Casasia chiapensis transferida a Radia matudae, 47:37-48.

Didymae

floribunda sp. nov., 44:73-80.

Elaeagia, México, primer registro 45:65-69.

uxpanapensis sp. nov., Oaxaca, 45: 65-69.

Psychotria

faxlucens sp. nov., Veracruz, 47:4964.

izabalensis subesp. oaxacana subesp. nov., Oaxaca, 47:49-64.

luteotoba sp. nov., Veracruz, 47:4964.

megalatha sp. nov., Oaxaca, $47: 49$ 64.

sousae sp. nov., Oaxaca 47:49-64.

veracruzensis sp. nov., Veracruz, 7:4964.

\section{Randia}

hidalgensis sp., nov., Querétaro, 7:3748.

lonicerioides sp. nov., Veracruz, 47:

37-48.

matudae nom. nov., Chiapas, 47:3748

pterocarpa sp. nov., Veracruz, 47:3748.

vazquezzii, sp. nov., Veracruz, 41: 37-48.
Richardia gandarae sp. nov., San Luis Potosí, 44:73-80.

Rutaceae

Casimiroa

calenderonii sp. nov., Oaxaca, 41:2326

ortografía correcta, calderoniae, 46: 89.

Decazyx

esparzae sp. nov., Tabasco, 43:1-3.

Rzedowskia véase Celastraceae

San Luis Potosí véase también (Agave;

Richardia gandaraé sp. nov.).

lluvia de polen, 46:53-74.

Savia véase Euphorbiaceae

Sciadodendron véase Arialiaceae

Scrophulariaceae

Kickxia

elatine ssp. crinita, en el Valle de

México, 41:27-32.

Lindernia

antipoda, México, nuevo registro 45:133-140.

Uroskinera hirtiflora var. breviloba var., nov., 45:133-140.

Scutellaria véase labiatae

Selaginella véase Pteridophyta

Semillas véase Ecofisiología

Setaria véase Gramineae

Sierra de Juárez véase Oaxaca

Sierra Madre del Sur

bosque mesófilo de montaña, fitogeografía, 44:97-102.

Sociedad Botánica de México

Boletín, índice acumulativo, 50:167-214

Solanaceae

Grabowskia

geniculata, número cromosómico, 45:141-142.

Lycium

estudios cromosómicos, 43:9-23. 
nuevas combinaciones y variedades, 43:5-8.

californicum var, interior var. nov., 43:5-8

leiospermum, número cromosómico, 48:145-146.

macrodon var. dipermum stat. et comb. nov., 43:5-8.

nodosum var. isthmense stat. et. comb. niov., 43:5-8.

parishi var. modestum stat. et. comb. nov., 43:5-8.

puberulum var. berberidoides stat. et. comb. nov. 43:5-8.

\section{Nicotiana}

glauca, ecología reproductiva, 41:4773.

Spigelia véase Loganiaceae

Spodoptera véase Lepidoptera

Stegnosperma véase Phytolaccaceae

Tabasco véase Decazyx esparzae sp. nov.; Luziola spruceana, nuevo registro; musgos

Tamaulipas véase también (Gleditzia, nuevo registro; $M$ imosa martindelcampoi sp. nov.)

bosque mesófilo de montaña, 48:139142

Tauschia véase Umbelliferae

Tehuacán-Cuicatlán (provincia florística)

fitogeografía, 50:00-00

vegetación arbórea, 45:49-64.

Teocelo véase Veracruz

tepescohuite véase Mimosa tenuiflora

Tetzcotzinco véase Texcoco

Texcoco véase México, Estado de

Tigridia véase Iridaceae

Tiliaceae

\section{Carpodiptera}

ameliae, México, distribución, 41: 166.
Triticale véase Gramineae

Tzonpotónic véase Hernández, Francisco

Umbelliferae

\section{Tauschia}

neglecta sp. nov., Estado de México, 41:19-21.

Uroskinera véase Scrophulariaceae

Valeriana véase Valerianaceae

Valerianaceae

Valeriana

vaginata, hojas descripción, 41:167170.

Valle de México véase México, Valle de Valle de la Muerte véase California Valle de Tehuacán-Cuicatlán véase Tehuacán-Cuicatlán (provincia florística)

Vegetación

acuática de lagos cráter véase Puebla arbórea véase Tehuacán-Cuicatlán insular véase Yucatán.

Venezuela véase Allosidastrum dolichophyllum sp. nov.

Veracruz véase también (Adiantum; Colubrina johnstonii sp. nov.; Psychotria faxlucens sp. nov.; Psychotria leutotoba sp. nov.; Psychotria veracruzenzis sp. nov.; Randia vazquezzii $\mathrm{sp}$. nov.; Rinorea uxpanapana sp. nov. Los Tuxtlas, especies CAM, 45:150153.

helechos, banco de esporas vease Pteridophyta.

Rubiaceae de, 50:43-68

Teocelo, bosque mesófilo, 48:35-63

Violaceae

Rinorea uxpanapana sp. nov., Veracruz, 45:133-140.

Yucatán véase también (Agave; musgos).

Vegetación insular, 45:23-37.

Yucca véase Agavaceae 
BOLETIN DE LA SOCIEDAD BOTANICA DE MEXICO Núm. 50, 1990

Zacate egipto véase Brachiaria mutica

Zacatecas véase también (Agave)

musgos, véase Bryophyta. 